\title{
On the Theory of Ion Noise in Microwave Tubes
}

\author{
Wallace M. Manheimer, Fellow, IEEE
}

\begin{abstract}
This work examines from a theoretical perspective recent experimental work regarding ion noise in the CPI continuous wave illuminator (CWI) and also earlier related ion measurements on a CW klystron. The measurements are to a large extent complementary, the former doing only microwave measurement, the latter doing only ion current measurements. The measurements are compared to theory which seems applicable. Many results do agree at least qualitatively with theory.
\end{abstract}

Index Terms-Ion noise, noise in microwave tubes, plasmas in microwave tubes.

\section{INTRODUCTION}

$\mathbf{T}$ HIS work examines theoretical aspects of ion noise in microwave tubes. It relies principally on measurements on a UHF tube, the EEV K376, and an X-band tube, the CPI Continuous Wave Illuminator (CWI). The former is documented in a Ph.D thesis by Smith [1] entitled "Ion Drainage in High Power Klystrons." I have documented this thesis at the National Technical Information Service in Springfield, VA, so that it can be more readily available. The work on ion noise in the CWI is documented in a series of notes and reports by Technology Service Corporation, a contractor for Naval Surface Warfare Center, Crane, IN, [2], [3]. Additional interpretation of ion noise in the CWI has been written up by Gilmour [4], a consultant to NSWC Crane. Furthermore, a publication summarizing this work is in preparation. Where appropriate, we denote this work as the Crane work and reproduce some of it here where it illuminates our theoretical developments.

Both Smith and the Crane team focus attention on ion effects, and thereby, noise near the carrier frequency. However, what they do are fairly different and to a large extent complementary. Smith does his experiments on a UHF klystron, the EEV type K376 which was manufactured by EEV, in Chelmsford, England. He calls the tube a valve; it generates $25 \mathrm{~kW}$ of power CW. Crane is concerned with the continuous wave illuminator (CWI), manufactured by CPI. This tube operates at $X$ band with a $\mathrm{CW}$ power of $10 \mathrm{~kW}$.

The ion noise (usually a slow phase oscillation or noise on the output at a hundred $\mathrm{Hz}$ to a few kilohertz) is important in the manufacture of the CWI tubes. Sometimes the tubes work fine, other units manufactured in apparently the same way have a great deal of ion noise. These noisy tubes have to be either redone or discarded. It is a significant cost and time

Manuscript received October 27, 1998; revised April 28, 1999. This work was supported by ONR.

The author is with Code 6707, Plasma Physics Division, National Research Laboratory, Washington, DC 20375-5000 (e-mail: manheime@ccsalpha2.nrl.navy.mil).

Publisher Item Identifier S 0093-3813(99)06931-3. driver for the tubes. The successful and unsuccessful tubes do not have significant measurable differences, and whatever the dividing line in parameter space is separating good from bad, the tubes apparently operate close to this boundary.

Below, some of the important parameters of both tubes are specified.

\section{EEV K376:}

- Frequency: UHF

- Beam Voltage: $17.5 \mathrm{kV}$

- Current: 4.6 A (Many of Smith's experiments were done at $3 \mathrm{~A}$ )

- $B=400$ Gauss (Beam has 16:1 area convergence, but only at most $4: 1$ field compression; mostly electrostatic focusing into the beam tunnel.

- Drift tube radius: $1.25 \mathrm{~cm}$, beam radius: $1 \mathrm{~cm}$

- Length of the tube: $88 \mathrm{~cm}$

- Typical pulse time: $1 \mathrm{~ms}$, although production tube runs $\mathrm{cw}$

- Gas pressure for Smith's experiments: $2 \times 10^{-7}-10^{-5}$ torr, although production tube runs at lower pressure.

- Smith modified the tube so that there is a little hole in the cathode to collect the ion current. This seems to be the only experiment where ion current was measured. In fact, Smith did only ion measurements, the tube did not have the microwave circuit in place. These modifications to the tube necessitated running at higher pressure than a production tube, although he could have run at pressures lower than $10^{7}$, but did not because typically not much would have happened in the ms pulse.

- The electron beam number density $\left(I / \pi r_{b}^{2}\right.$ ev) is about $6 \times 10^{8} \mathrm{~cm}^{-3}$, while the gas density varies from $6 \times$ $10^{9}$ to $3 \times 10^{11} \mathrm{~cm}^{-3}$ as the pressure increases from 2 $\times 10^{-7}$ to $10^{-5}$ torr. Thus there is more than enough gas to fully neutralize the beam if it is sufficiently ionized and the secondary electrons generated escape. It does not seem as though one has to worry about neutral resupply or recycle to explain Smith's results.

\section{The CPI CWI:}

- Frequency: $X$ Band

- Beam voltage: $20 \mathrm{kV}$

- Beam current: 3A

- Magnetic field: 2.6 kG. Gun has 45:1 area ratio compression into beam tunnel, mostly electrostatic. The field for Brillouin flow is $1.1 \mathrm{kG}$.

- Drift tube radius: $1.5 \mathrm{~mm}$, beam radius: $1 \mathrm{~mm}$

- Length of tube: About $20 \mathrm{~cm}$

- Pulse time: CW 
- The diagnostics in the Crane work are entirely microwave diagnostics, there are no ion measurements.

- Gas pressure: Ion pumps near the collector and gun read a pressure of about $10^{-8}$ torr when the tube is operating. This pressure apparently varies little from quiet to noisy tubes.

- The beam number density is $7 \times 10^{10} \mathrm{~cm}^{-3}$, while the background density at $10^{-8}$ torr is only $3 \times 10^{8} \mathrm{~cm}^{-3}$. Thus even if the background is fully ionized, it cannot provide nearly sufficient ions to give the noise which is observed. Thus for at least the noisy tubes, resupplying and recycling of neutrals from the walls has to be important.

As is apparent, Smith's and the Crane work complement each other nicely from a scientific point of view. The former used a pulsed system at high pressure and did ion diagnostics, the latter used a CW system at low pressure and did microwave diagnostics.

All workers emphasize the relation of ion noise to the scalloping of the electron beam. A beam which is not perfectly matched into the microwave circuit has ripples on its envelop. As parameters of the tube vary, these could affect the scalloping in ways that can be relatively easily specified (for instance slight variations in the magnetic field) or in ways that cannot be so easily specified (for instance ions in the tube or focusing regions of the gun could affect the focusing dynamics of the beam, and thereby the scalloping).

The Crane team has accumulated a great deal of data on the properties of the noise as a function of many possible variables in the tube. They reported tests of four tubes [2]-[4]. The first showed no noise no matter how the parameters were varied. The second showed no noise at the manufacturer's specified parameters, which they called the name plate value (NPV), but showed some noise at other parameters. The third tube showed noise at most parameter values, but the noise could be sometimes tuned on and off by varying parameters, and the fourth tube showed noise no matter what. Most of the results they showed were for the third tube, which allowed variation in noise to be most easily studied. The parameter which had the most effect on the nose was the solenoid current, the second most important parameter was the beam voltage, the third most important was the cathode filament current, other parameters had little effect on the noise. The Crane group concluded that the effect of the filament current was not due to stray magnetic fields, because the noise took some time to react to the solenoid current variation, whereas the magnetic field variation would of course occur instantaneously.

The noise is typically observed as a slow variation of the output phase with time. Shown in Fig. 1 is a plot (from [2]) of output phase versus time for the CWI at five different solenoid currents (the NPV current is about $20 \mathrm{~A}$ ). There are several comments regarding Fig. 1 and the other figures like it. First, the low amplitude (phase change of about 0.1 degrees), fast (frequency about $1 \mathrm{kHz}$ ) oscillation is instrumental and not a characteristic of the microwave tube. This high frequency, in fact is a multiple of $60 \mathrm{~Hz}$. Second, the lines which are perfectly flat have been time averaged, so they actually look like the other lines shown with the small oscillation [2], [5].

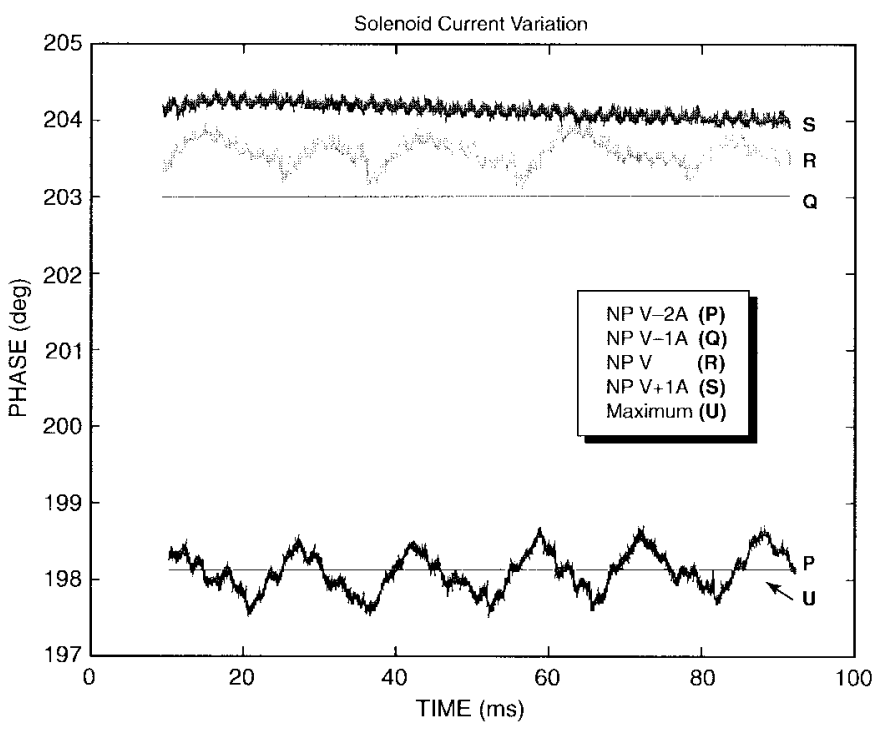

Fig. 1. Output phase of the CWI as a function of time for five different solenoid currents.

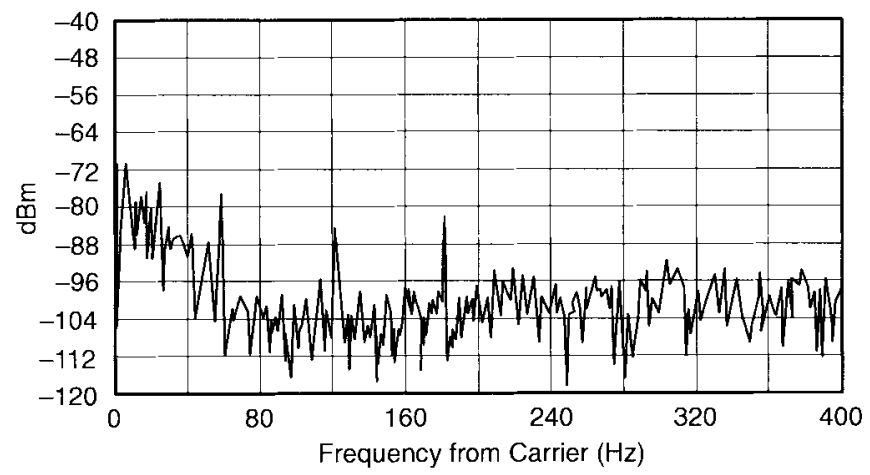

(a)

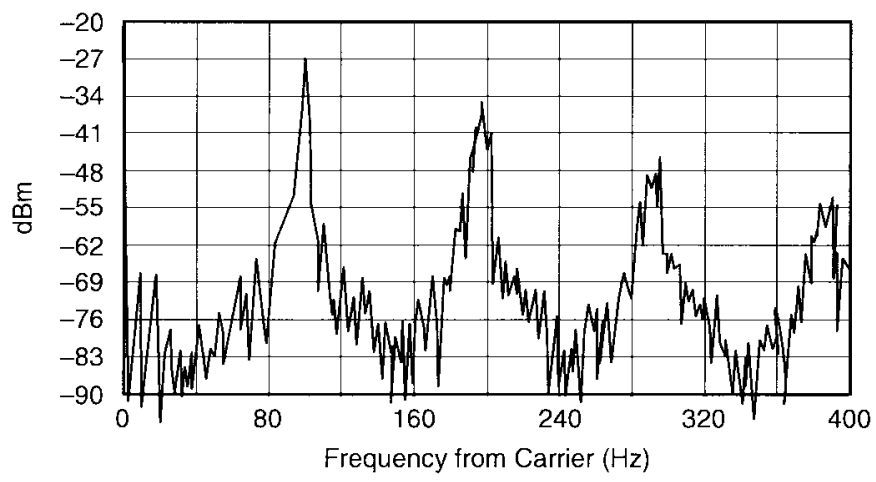

(b)

Fig. 2. Phase spectrum of the CWI at two different magnetic fields $1 \%$ apart.

Noisy tubes show a phase variation of half a degree or so with about a $10 \mathrm{~ms}$ period. However the noise can be tuned in and out by optimizing the solenoid current; at one amp above the minimum current, and at the maximum current the tube shows little noise, but there is noise in between. Another way to view this is in frequency space. Shown in Fig. 2 is a plot (from [4]) of the spectrum in $\mathrm{Hz}$ away from the carrier frequency for two different magnetic fields, now only apart by $1 \%$. 


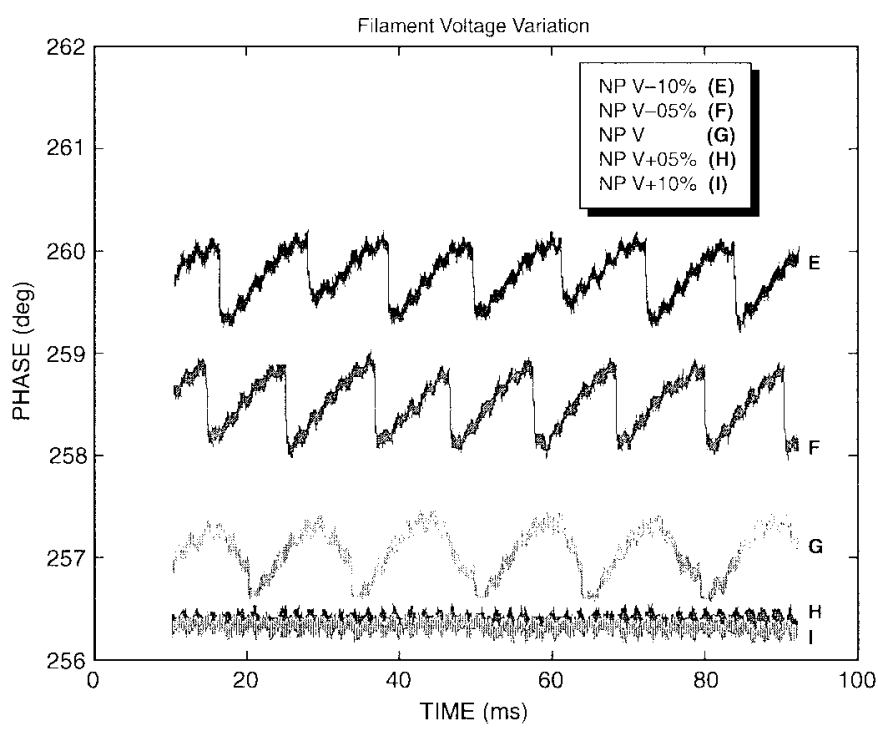

Fig. 3. Output phase of the CWI as a function of time for different cathode filament currents.

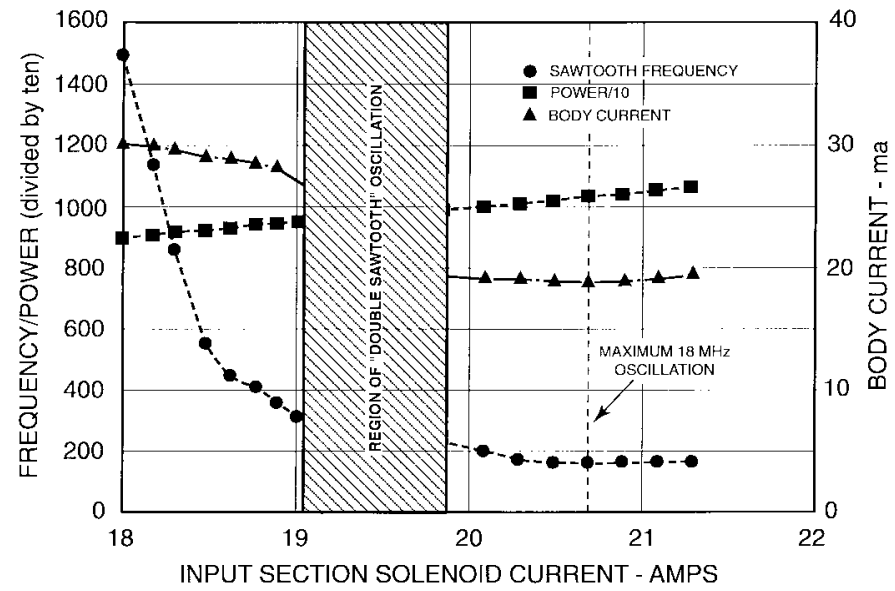

Fig. 4. Sawtooth frequency, body current, and microwave output power as a function input solenoid current.

The phase curves in Fig. 1 are rather smooth function of time; however other data shows that the phase dependence can appear more like a relaxation oscillation. This is most apparent in the Crane work in their studies of the dependence of phase on cathode filament current. Shown in Fig. 3 are plots of phase as a function of time at different filament currents. Clearly, at some currents there is a very sharp drop in phase, giving the curve the appearance more of a sawtooth than a smooth curve. The phase frequency is typically $100 \mathrm{~Hz}$, although there is some variation. The Crane team has studied frequency as a function of the current of the input solenoid, and this is shown in Fig. 4. At the lowest current, the frequency is above $1 \mathrm{kHz}$, but as the current is increased, it drops to its more normal values of $100-200 \mathrm{~Hz}$. There is a range of current where there is a double sawtooth structure to the phase oscillation, and when passing through this range, there is a rather abrupt drop in body current.

It seems to this author that variations in the gas pressure in the tube may be playing a role despite the lack of variation of signals on the ion pumps. The pumps are quite far away, and the volume of the circuit region is very small compared to the volume near the pumping ports. Also, there may be different parts of the wall, in the vicinity of the microwave circuit, that alternately emit and absorb gas, and these may be different from tube to tube. This emission and absorption could all be locally determined, so that there would be very little signature of it at the ion pumps. In any case, it seems useful to record this speculation here, and also make the obvious suggestion; namely that if there is any way the pressure in the operating tube could be measured in the region of the microwave circuit and focused beam, it would probably be worth the resources to do so.

For the most part, this report concentrates on the theoretical aspects of the ion noise, and more generally, on the behavior of the ions and the plasma produced in the tube. In all cases the tube will be assumed uniformly magnetized. However, there are a number of different axial electrostatic potential profiles that might exist in the tube. These are sketched in Fig. 5(a)-(c). Fig. 5(a) is the normal axial potential profile one might expect in a microwave tube. The cathode is at some large negative potential. The tube wall is grounded, but the region of the beam is at a lower potential because of the beam space charge, and finally, the collector is grounded. Ions produced by the beam are electrostatically confined to the beam region by the negative charge of the beam as long as the ion density is less than the beam density. However, the magnetic field is sufficiently weak that the ions are essentially unmagnetized even though the electrons are strongly magnetized. The ions then drain toward the cathode and are repelled by the anode. This is generally the configuration examined by Smith, and the theory developed here seems to give a reasonably good model for the observations.

Fig. 5(b) shows the potential profile if the beam profile is scalloped. The scallops correspond to potential wells which axially alternately confine the ions and electrons. As the plasma is formed, the wells begin to fill up. However, as they fill, the potential profiles modify due to the trapped particles until at some point the wells disappear. At this point the ions will discharge to the cathode as they are produced. This variation in ion current will also affect the focusing of the beam and the associated ripple generation. The relaxation oscillation of the phase of the microwave signal is generally interpreted as a signature of this phenomenon. As the ions and electrons build up in the traps, the output phase slowly changes due to, for instance, slight changes in say beam velocity. Then when the traps dump, the phase switches, possibly abruptly, back to its initial value. If the ions are trapped above the potential maxima and electrons below the potential minimums, we will see that theory predicts a sensitivity to the magnetic field and voltage which seems to be reasonably well obeyed. Furthermore, theory gives reasonable estimates for the periods of these oscillations.

Fig. 5(c) shows the potential profile with a depressed collector. This might be used in a microwave tube to increase efficiency. It is in fact used in the CPI CWI, so it is actually a combination of Figs. 5(b) and (c). The reason Fig. 5(c) may be very different is that the potential structure as shown is 


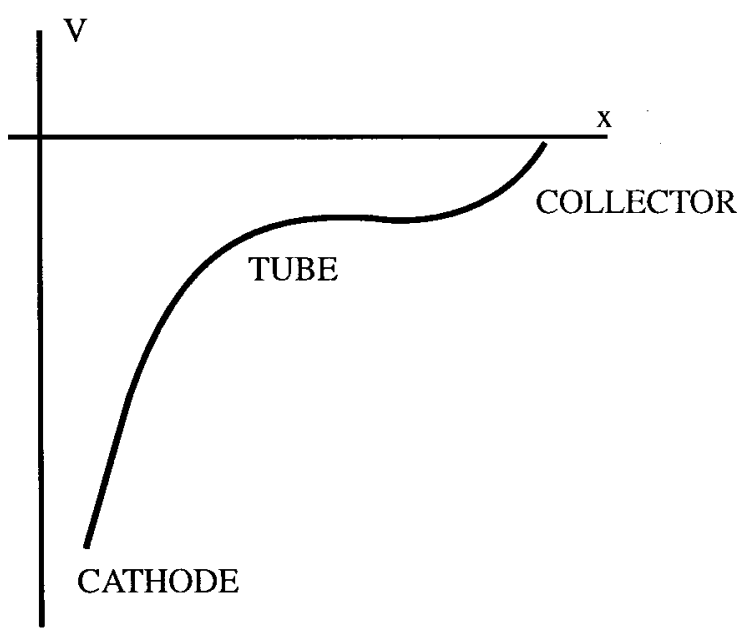

(a)

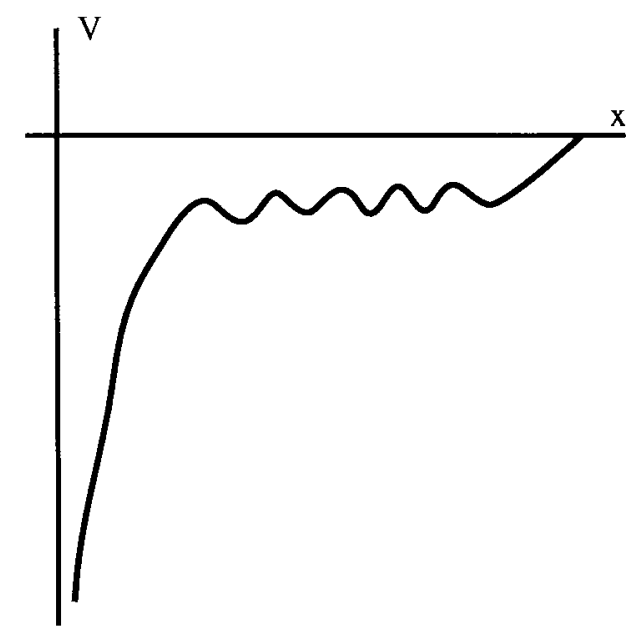

(b)

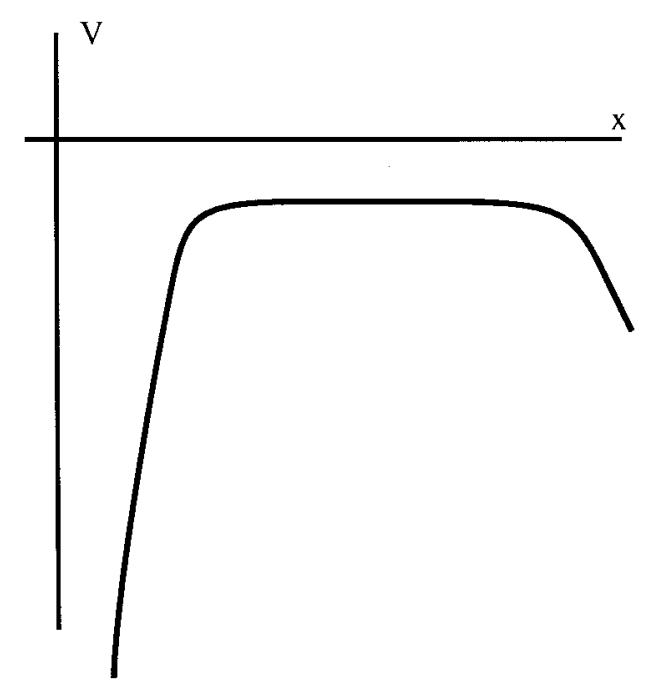

(c)

Fig. 5. Possible axial potentials in a linear microwave tube.

actually a Penning trap. These are used to confine electrons, and actually confine them extremely well. The reason is that electrons in the Penning trap can be a thermal equilibrium, and as such can be confined for very long times. The theory of Penning traps would lead one to believe that a tube with a depressed collector could contain a large density of trapped electrons. However, in the CWI, the fact that the magnetic field lines threading the beam may strike the wall before going into the depressed collector has a large effect on the number of trapped electrons. Taking this into account one seems able to explain the results of the Crane experiments on the effect of the depressed collector. This leads to an obvious speculation, namely that in a $\mathrm{CW}$ gyrotron operating with a depressed collector, where the voltages and magnetic field strengths are considerably higher, there could be many more trapped secondary electrons.

This work is organized as follows. First, Section II discusses the microwave phase shift as a function of the axial profile of the electrostatic potential in the microwave tube. Section III discusses electron production by the secondary electrons as contrast to production directly by the beam. Then we discusses the physics of the various traps. Section IV discusses that of Fig. 5(a), and so on to Section VI which discusses that of Fig. 5(c). One conclusion, especially with regards to Fig. 5(b) and possibly Fig. 5(c) is that ion production from the secondary electrons is very important, because the secondaries themselves form a confined plasma with particle energy comparable to that at the maximum of the ionization cross section. Section VII very briefly discusses the question of gas supply in the CWI, Section VIII then discusses what directions future theoretical studies go in, and Section IX summarizes conclusions. There are two appendices which present more detailed calculations of ionization from secondaries and calculations of equilibria.

\section{The MicrowaVe Phase ShIFT}

Here, we discuss the microwave phase shift, as has been done elsewhere [6]. The key is that the CWI is a traveling wave amplifier. The simplest model for traveling wave amplification is that two waves, one traveling with the beam velocity $v_{z}$ and one traveling with the phase velocity in the slow wave circuit $v_{p h}$, couple with coefficient $\alpha$ so that the dispersion relation for the system is

$$
\left(\omega-k v_{z}\right)\left(\omega-k v_{\mathrm{ph}}\right)=-\alpha^{2}
$$

which gives wave number

$$
k=\frac{\left(v_{z}+v_{\mathrm{ph}}\right) \omega \pm \sqrt{\omega^{2}\left(v_{z}-v_{\mathrm{ph}}\right)^{2}-4 v_{z} v_{\mathrm{ph}} \alpha^{2}}}{2 v_{z} v_{\mathrm{ph}}} .
$$

Note that the fast and slow space charge waves on the beam are assumed to be nondegenerate. The reason is that for the CWI, the beam plasma frequency is not small compared to either the temporal growth rate, or even the microwave frequency. If $v_{z}$ is nearly equal to $v_{\mathrm{ph}}$, the term in the radical gives the amplification. However, here we are more concerned with the phase, the term outside the radical. For maximum amplification, where $v_{z}=v_{\mathbf{p h}}$, the total phase length is $\varphi=\omega L / v_{z}$. Taking $\omega=6 \times 10^{10}$ as is characteristic of an $X$-band microwave tube, and other parameters of the CWI, we find a phase length of about 150 radians, or about 9000 degrees. Now let us consider what happens when the beam 


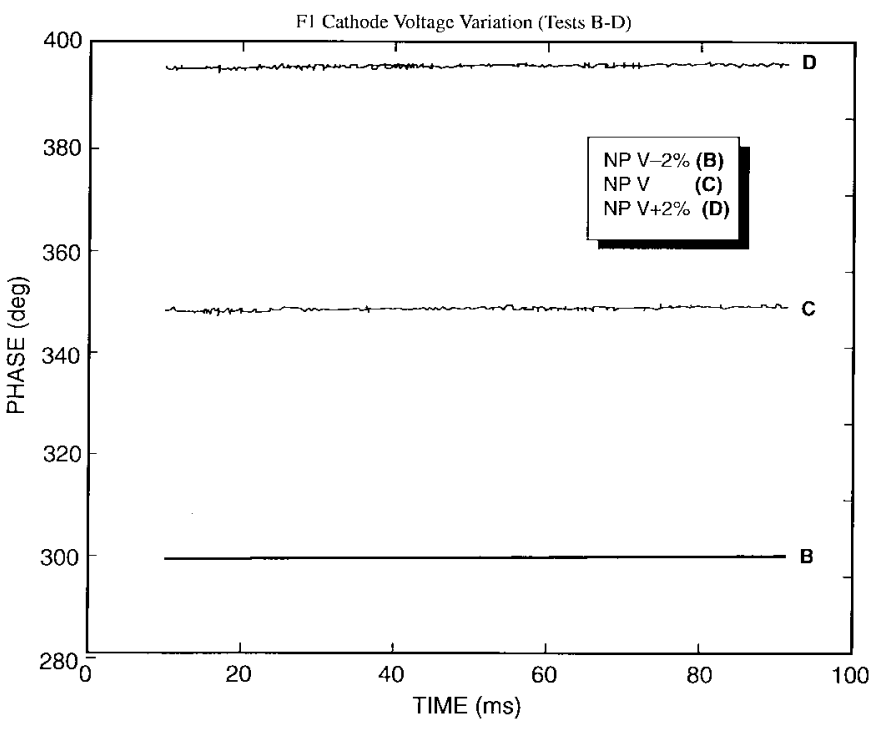

Fig. 6. Phase shift in the CWI as a function of beam voltage.

velocity changes due to a change in voltage. Since the beam velocity varies, but the phase velocity does not, we find

$$
\Delta \varphi=-\frac{\omega L \Delta v_{z}}{2 v_{z}}
$$

where we have assumed that $v_{z} \approx v_{\mathrm{ph}}$. Thus a $2 \%$ change in voltage, which gives a $1 \%$ change in velocity, will give a $1 / 2 \%$ change in phase.

Experiments in Crane, reproduced in Fig. 6 show clearly that a $2 \%$ change in voltage causes a $1 / 2 \%$ change in phase, as predicted by (2). Furthermore, as Fig. 6 demonstrates, the sign of the phase plotted is actually a mirror image of the phase as defined in (2); lower velocity (i.e., lower voltage) clearly has to give a higher phase.

Now envision the case where the potential has a slight variation along the tube axis. Let us assume that because of a ripple in the beam envelop, there is a sinusoidal potential variation along $z$ given by $\phi_{o} \cos \left(k_{B} z+\eta\right)$, where $k_{B}$ is the ripple wave number, generally equal to the cyclotron wave number $\Omega_{c} / v_{z}, \Omega_{c}$ being the cyclotron frequency. Then, if $\phi_{o}$ is small, we have approximately

$$
\varphi \approx \frac{\omega L}{v_{z}}-\frac{\omega e \phi_{o}}{2 m v_{z 0}^{3} k_{B}}\left\{\sin \left(k_{B} L+\eta\right)-\sin \eta\right\} .
$$

We see from (3a) that if $k_{B} L=2 n \pi$, the phase fluctuation due to the ripple will cancel out. For the CWI, with a 20 $\mathrm{kV}$ beam and a $2.3 \mathrm{kG}$ field, the ripple wavelength is about a centimeter and a quarter, so there are about 16 cyclotron wavelengths in the $20 \mathrm{~cm}$ length. Thus a change in $B$ of $6 \%$ (corresponding to a change of solenoid current of this amount) will change the total number of ripple wavelengths by one. Hence a $3 \%$ change will vary the phase shift from maximum to minimum, and the phase shift should be rather sensitive to a $1 \%$ change in solenoid current. As regards the voltage, the velocity goes as the square root of the voltage, so $k_{B}$ is half as sensitive to changes in voltage as to magnetic field changes. This also seems to be consistent with the measurements, as has been pointed out.
We have assumed the ripple in beam envelop to be purely sinusoidal. Actually velocity spread on the beam can cause the downstream ripples to wash out by phase mixing. In the CWI, a $6 \%$ velocity spread should be enough to phase mix the last ripple. If we model the phase mixing with exponential damping for simplicity, we find that if the length $L$ is large compared to the damping length

$$
\varphi=\frac{\omega L}{v_{z}}+\frac{\omega e \phi_{O}}{2 m v_{z o}^{3} k_{B}} \sin \eta
$$

so the phase shift depends mostly on the input conditions. Notice that the maximum of the factor in the brackets in (3a) is two, whereas the maximum value of $\sin \eta$ in (3b) is unity. Thus the maximum phase departure for a beam with no velocity spread is about twice as great as that for a beam with sufficient velocity spread. Thus a small amount of velocity spread may actually be beneficial as regards ion noise (although obviously it should be small enough so as not to adversely affect the microwave generation).

Hence the following possible picture emerges for the phase shift in the CWI. The beam has a ripple which gives rise to phase shift. As the background gas is ionized, the ions trap above the potential minimums, and the electrons trap below the potential maxima. As their densities build up, this reduces the peak to peak potential. When it gets sufficiently small (or zero), the traps dump their particles and the process starts anew.

If this trapping and untrapping causes the potential amplitude $\phi_{0}$ to oscillate between zero and its maximum value, the phase oscillation should have a maximum peak to peak amplitude of a quarter of a degree according to (3a) and an eighth of a degree according to (3b). The former agrees qualitatively, but is somewhat less than what is measured. The latter is definitely less than what is measured. It may be that the ripple depth is greater than $10 \%$, especially at the input. Also it might be that the ripple traps are not equally effective in trapping electrons and ions.

\section{IONIZATION FROM SECONDARIES}

We call a beam electron a primary electron and it ionizes the background gas to some extent. The electrons that it directly produces we call secondaries. The cross section for ionization by electron impact is given for a number of gases is and is shown in Fig. 7, taken from Brown [7]. At high energy, the cross section has a dependence on energy typically proportional to $\ln E / E$. An approximate expression for cross section, reasonably accurate for most gases between about a kilovolt and several tens of kilovolts, is

$$
\sigma_{i}\left(\mathrm{~cm}^{2}\right)=\frac{3 \times 10^{-17} \ln [23 V /(\mathrm{keV})]}{V(\mathrm{keV})} .
$$

As is apparent from Fig. 6, the cross section has a broad maximum, above $2 \times 10^{-16} \mathrm{~cm}^{2}$, for incident electron energies between about 40 and $500 \mathrm{eV}$. The primary, which has energy of perhaps $20 \mathrm{keV}$, on the other hand, produces ionization with a cross section of about $5 \times 10^{-18} \mathrm{~cm}^{2}$. Thus it is natural to think that in some circumstances the secondaries 


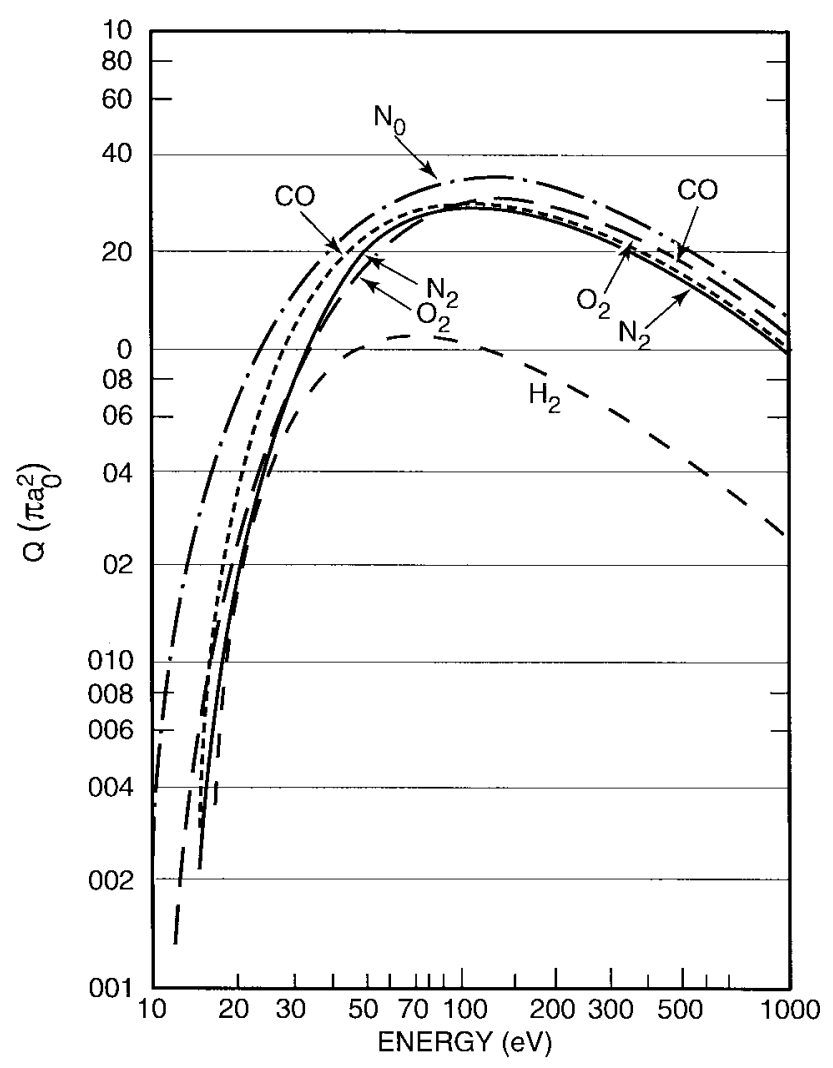

Fig. 7. Ionization cross section as a function of energy for various gases from Brown. $\pi a_{0}^{2}=.88 \times 10^{-16} \mathrm{~cm}^{2}$.

could give rise to more ionization than the primaries. In fact, in normal electron propagation in say air or a plasma, this additional ionization typically gives about triple the beam ionization [8], [9]. In a microwave tube, where the background pressure is very low, the ionization by the secondaries may be greatly reduced because they may not be confined long enough to give significant additional ionization. However, in other instances the secondaries may be confined [as in for instance Fig. 5(b) and (c)], and in this case, the ionization from the secondaries could be an important factor. Alternatively, the density of the unconfined secondaries may be large enough that further ionization by them could be important. Here we consider both possibilities.

Consider a beam electron of energy $E$. As it propagates through the microwave tube of length $\mathrm{L}$, which contains a background gas of density $N\left[N\left(\mathrm{~cm}^{-3}\right)=3.5 \times 10^{16} P\right.$ (torr)], it produces secondary electrons at a rate

$$
\frac{d n}{d t}=N \sigma_{i} v_{b} n_{b}
$$

where $v_{b}$ and $n_{b}$ are the beam velocity and density. Furthermore, the number of secondaries each beam electron produces is $\zeta=N \sigma_{i} L$ where $L$ is the length. For the CWI, with $L=20$ and $E=20 \mathrm{keV}$, we find $\xi=3 P$. Thus if the pressure is $10-7$ torr, each electron produces only about $3 \times 10^{-7}$ secondary electrons and ions. Since each secondary electron is ultimately collected somewhere, on the radial wall or the collector, the current from the secondaries is only about a microamp. Notice that this is very small compared to the body current, typically
$30 \mathrm{~mA}$ from Fig. 4, so that it is very unlikely that this current would be observed in a current measurement.

Now consider the secondary, and ask the rate at which it produces ionization. The formula is the same as for primaries, except that the energy is the energy of the secondary. Since $\sigma$ vat an energy of $100 \mathrm{eV}$ is greater than the value at $20 \mathrm{keV}$, the production rate from secondaries can be larger that the production rate from beam electrons even if the density of the secondaries is smaller than the beam density.

We consider two possibilities for production by confined secondaries. First assume that the electron is produced in a zero field region and is confined by a steep potential gradient at each end. Then the generation of tertiaries is governed entirely by the energy spectrum of the secondaries. Typically a small fraction of secondaries (perhaps 20\%) have sufficient energy to further ionize the gas. As these slow up, they and their progeny generate additional electrons. Standard calculations show that each secondary generates typically two to four additional electrons. A qualitative example of such a calculation is sketched out in Appendix A.

The second possibility is that the secondary is produced in a region of non zero electric field, but is confined to a potential well. In this case, the secondary can produce additional ionization if it is born at high enough potential energy, even if it is born with zero kinetic energy. Since ripples in the beam envelop usually generate potential wells of over 100 volts (near the maximum of the ionization cross section), almost all electrons will be able to ionize the background. Then it is simplest and probably most accurate to consider the trapped electron as a fluid with density $n$. In this case the density builds up as

$$
\frac{d n}{d t}=\frac{I \sigma_{i}(E) N}{\pi e r_{b}^{2}}+\left\langle\sigma_{i}(t) V_{t}\right\rangle n N
$$

where $I$ is the beam current, $r_{b}$ the beam radius and $\left\langle\sigma_{i}(t) V_{i}\right\rangle$ is for the trapped electrons, assumed to be at energy about 100 $\mathrm{eV}$. Thus $\sigma V$ for the confined electrons is about $1.3 \times 10^{-7}$ $\mathrm{cm}^{3} / \mathrm{s}$. This is a rather large value, and since the ionization rate is proportional to $n$ can give rise to exponential increase in ion density, that is avalanche ionization. Thus, theory shows that the effect of ionization by secondaries can be either relatively unimportant, if they are unconfined, and if their density is much less than the beam density; or quite important if they are confined.

\section{The Normal Axial Potential Profile}

\section{A. The Ion Current}

Fig. 5(a) plots the potential along the axis of the microwave tube for a beam with no ripple. Although the wall of the microwave circuit is grounded, Fig. 5(a) shows the potential along the axis as depressed to a negative value. This is due to the space charge of the beam. The electron number density of the electron beam is given by

$$
\begin{aligned}
n_{b}= & I /\left(\pi r_{b}^{2} e v_{b}\right)=3.4 \times 10^{10} \mathrm{~cm}^{-3} I(\text { Amps }) / \\
& {\left[V_{o}^{1 / 2}(\text { volts }) r_{b}^{2}(\mathrm{~cm})\right] }
\end{aligned}
$$


where $v_{b}$ is the beam velocity and $V_{o}$ is the beam voltage. Assuming uniform charge density in the beam, Poisson's equation gives the potential between the center and edge of the beam $V_{s}$ as

$$
V_{s}(\text { volts })=1.5 \times 10^{4} I(\mathrm{Amps}) / V_{o}^{1 / 2}(\text { volts }) .
$$

Since the beam at radius $r_{b}$ might not fill the drift tube at radius $a>r_{b}$, the potential drop between the grounded drift tube wall and beam center is given by

$$
V_{b}=-V_{s}\left[1+2 \ln \left(a / r_{b}\right)\right] \text {. }
$$

While the above formulas are in mks units, it is also worth noting some results from cgs units, namely that 300 volts $=1$ statvolt, and the charge and current in cgs are $3 \times 10^{9}$ times larger than they are in mks units. Furthermore, in cgs units, voltage and charge per unit length have the same units, and for the beam in cgs units, the relation between charge per unit length $\Lambda$, and beam voltage $V_{s}$ is simply

$$
\Lambda=V_{s}
$$

For an axial potential of the form of Fig 5(a), as ions and secondary electrons are produced by the beam electrons, the secondary electrons drain to the collector and the ions drain to the cathode. Even though the potential drop to the collector is so much less than it is to the cathode, the electrons are so much lighter that neglecting their space charge is a reasonable approximation.

We now give an approximate calculation relating ion current to the other parameters of the tube. A more accurate calculation will be given in Appendix B. The equation for number conservation equation for the ions in steady state, integrated over the tube cross section, (calculations here will generally be presented in cgs units) is

$$
\frac{\partial}{\partial x} \Lambda_{i} V_{i}=I \sigma_{i} N
$$

where $x$ is the coordinate along the axis of the tube, $V_{i}$ is the ion velocity, $\sigma_{i}$ is the effective ionization cross section, and $N$ is the volumetric number density of the background gas. Since none of the secondaries are confined, we assume the ionization cross section given by (4) and Fig. 7. We will discuss this approximation shortly.

Integrating (8) over the length of the tube, assuming the right-hand side is homogeneous, and there is no ion current to the collector, we find

$$
\left.\Lambda_{i} V_{i}\right|_{\text {edge }}=I \sigma_{i} N d
$$

However, the velocity at the plasma edge is related to the axial voltage drop across the beam, which, by (9) and (10) is related to the difference in ion line charge density from one end of the beam to the other. (Note that the space charge density from the electron beam is uniform axially, and the space charge from the secondary electrons has been neglected.) Thus $\left.V_{i}\right|_{\text {edge }}=\left[2 e V_{a x} / M\right]^{1 / 2}$ and

$$
V_{a x}=K\left[\Lambda_{o}-\Lambda_{\text {edge }}\right]
$$

where $K=1+2 \ln \left(a / r_{b}\right)$, and $\Lambda_{0}$ is the ion line space charge density near the collector. We make the reasonable assumption, and show in Appendix $\mathrm{B}$, that $\Lambda_{\text {edge }}=\Lambda_{\circ} / 2$. This then gives the steady state relation for line charge density as a function of beam current and ionization rate as

$$
\Lambda_{o}^{1.5}=\frac{2 I \sigma_{i} N d}{\left[\frac{K e}{M}\right]^{1 / 2}} .
$$

Now consider the secondary electrons. Since they are generated at the same rate as the ions, but escape with a velocity $[\mathrm{M} / \mathrm{m}]^{1 / 2}$ times greater, their density should be $[\mathrm{m} / \mathrm{M}]^{1 / 2}$ times less than the ion density. Typically we assume that the ion density is much less than the beam density. If this be the case, ionization by the secondaries is small compared to ionization by the beam electrons. If the ion density is equal to the beam density, the highest density at which the ions can be radially confined, then, depending on mass ratio and beam energy, the ionization by the secondaries can be comparable to or perhaps even larger than the beam ionization.

For the parameters of the EEV type K376, and assuming $\mathrm{M}$ is 30 times the proton mass (Smith thinks the most likely components are $\mathrm{CO}$ and air) we find that

$$
\Lambda_{o}(\mathrm{coul} / \mathrm{cm})=5 \times 10^{-7} P^{2 / 3} \text { (torr) }
$$

and the axial voltage drop across the tube is

$$
\left.V_{a x}(\text { volts })=4.2 \times 10^{5} P^{2 / 3} \text { ( torr }\right) .
$$

The linear charge density of the electron beam in Smith's experiments is typically $3 \times 10^{-10}$ Coulombs per cm. Fig. 8 shows Smith's curve of ion charge as a function of pressure. It is measured by taking the steady state ion current and multiplying by the time to form steady state. The curve is almost certainly incorrect, at least as far as the vertical axis is concerned. For instance at a pressure of $10^{-6}$ torr, it shows an ion charge of almost $10^{-6}$ coulombs, whereas the charge in the beam is only about $3 \times 10^{-8}$ coulombs in the $88 \mathrm{~cm}$ beam tube, about a factor of 30 lower. Clearly if the ion charge density is equal to that of the beam, the beam cannot be confined, many ions escape radially, and the model breaks down. One possible interpretation of Fig. 8 is that ions are stored in many other places besides within the microwave circuit; another interpretation is that the labeling of the vertical axis of Fig. 8 is incorrect. Perhaps a less ambiguous interpretation is to look at the break in the experimental curve and interpret it at the point where the ion charge density is equal to that of the beam. Smith also makes this interpretation. The theoretical result for the parameters of Smith's beam is that $\Lambda_{0}=$ beam linear charge density when $[P]^{2 / 3}=5 \times 10^{-4}$. The predicted pressure is about $1.1 \times 10^{-5}$, not too far from the measured pressure of about $7 \times 10^{-6}$.

Another check comes from the steady state current measurements. For a pressure of $10^{-6}$ torr, a rather typical pressure for Smith's experiments, we find the voltage drop across the tube axially is 42 volts. For an ion with a mass of 30 times the proton mass, this is a velocity of $1.2 \times 10^{6} \mathrm{~cm} / \mathrm{s}$, and an ion drainage current to the cathode of about of $40 \mu \mathrm{Amps}$ from (12). A plot of Smith's measured current as a function of pressure is shown in Fig. 9. The predicted current is within 


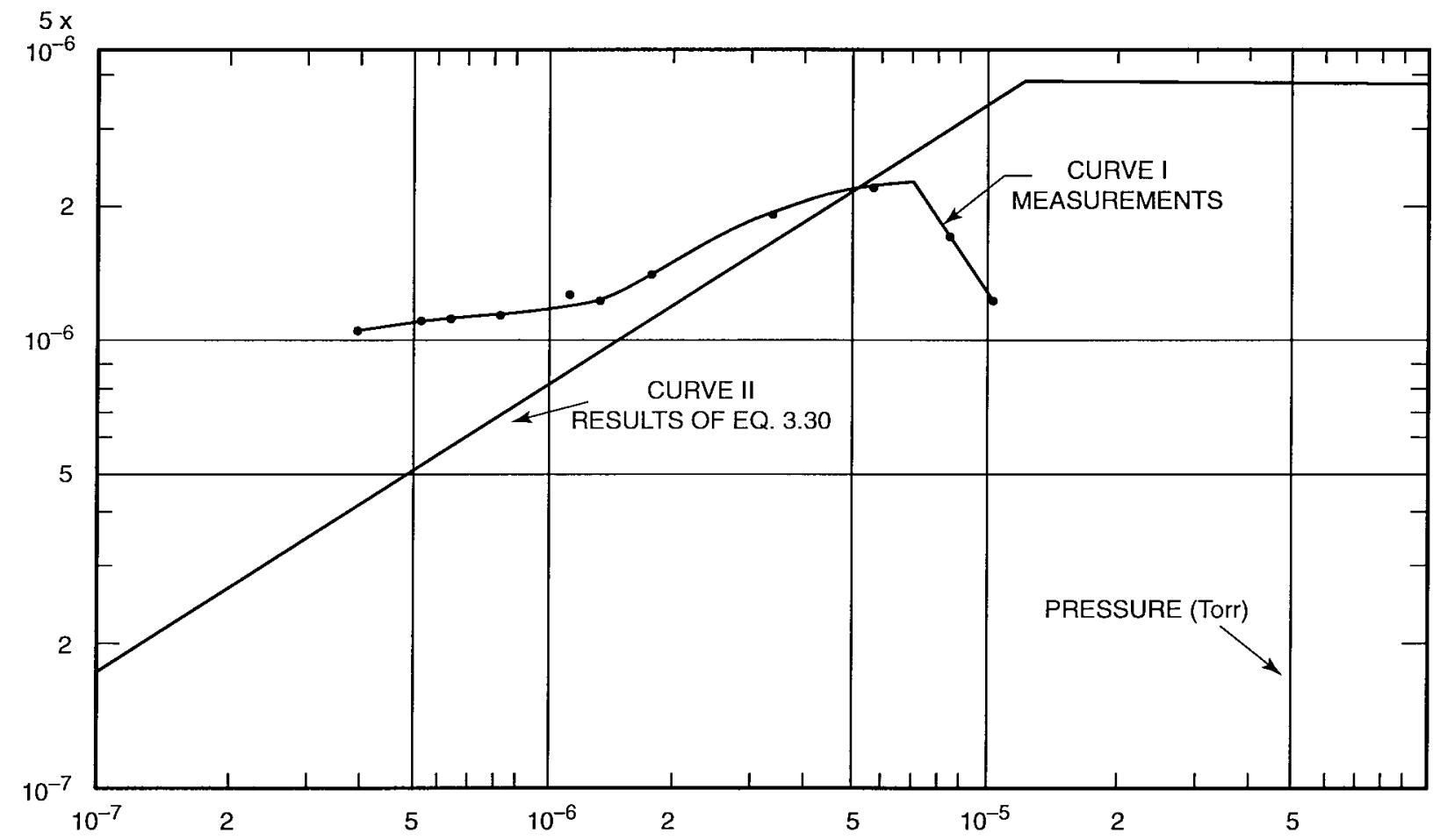

Fig. 8. Plot of ion charge as a function of gas pressure from [1].

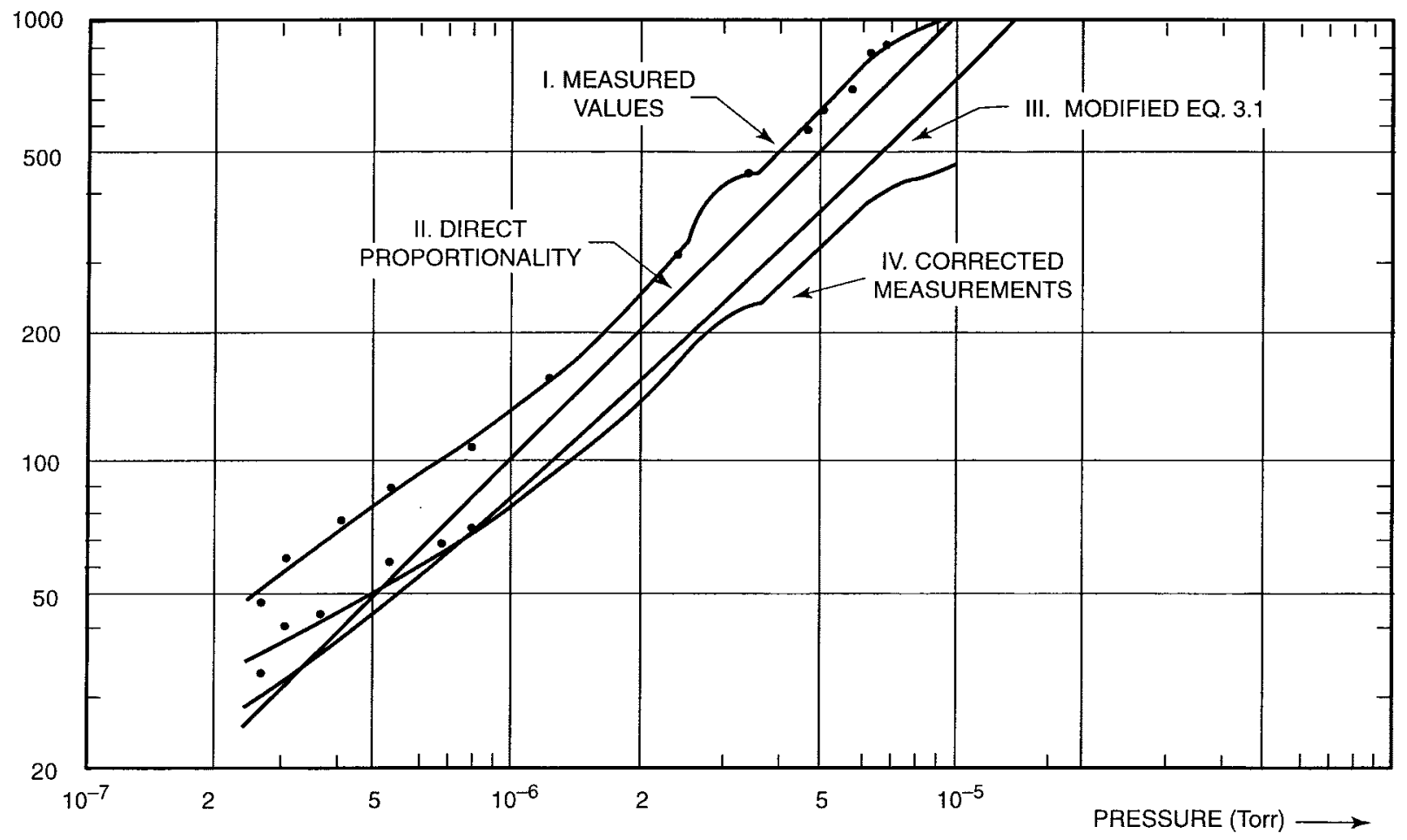

Fig. 9. Plot of ion current as a function of pressure from [1].

a factor of two of Smith's corrected measurements. If there is additional ionization due to for instance trapped secondary electrons, this might explain the difference.

Inherent in our calculation of the effect of secondaries is the assumption that the mass is 30 times the proton mass. If the ion mass could be independently identified, for instance by a mass spectrometer behind the cathode, it would tell a great deal about the different constituents of the ion stream to the cathode. The current is predicted to be nearly independent of ion mass. However, the ion generated voltage drop and the ion 
charge density (and also the charge density at which the ion density equals the beam density) does depend on ion mass. Smith's measurements do seem to indicate that the gas more likely has mass of about 30, and is less likely to be either protons or heavy metal ions. Of course a measurement with a mass spectrometer would be the ultimate test.

For the CWI, we take $d=10 \mathrm{~cm}$, accounting for the fact that ions can exit both ends, assume the ions are protons, as is characteristic in high vacuum systems, and find

$$
\begin{aligned}
\Lambda_{o}(\mathrm{coul} / \mathrm{cm}) & =3 \times 10^{-8} P^{2 / 3}(\text { torr }) \\
V_{a x}(\text { volts }) & =2.5 \times 10^{4} P^{2 / 3}(\text { torr }) .
\end{aligned}
$$

\section{THE SCALLOPED BEAM}

In Section IV we discussed ion currents in a beam where the axial potential is smooth. Actually the beam may have scallops in its envelop, and this could give rise to an axial electrostatic potential like that shown in Fig. 5(b). If the beam radius has an axial profile $r_{b}(z)=\epsilon \cos k_{z}$, then the potential along the axis from (9) is

$$
V_{b}(z)=V_{b}+2 V_{s} \epsilon / r_{b} \cos k_{z} .
$$

This potential alternately can trap ions and electrons about positions of potential minimums and maxima, respectively. Notice that these traps overlap in space, so trapped electrons and ions coexist at the same spatial positions, even though they are trapped in different traps. Let us then calculate the maximum trapped energy for a trapped particle. Clearly it is $4 V_{s}\left(\epsilon / r_{b}\right)$. If we assume a canonical value of $10 \%$ for $\epsilon / r_{b}$, then for either the CPI CWI or EEV K376, the maximum trapped energy is about $150 \mathrm{eV}$. As the background gas is ionized, the traps fill up with an excess of ions above the potential minimums and an excess of electrons below the potential maxima. However, as ions accumulate around the potential minimums, the potential increases according to (10) (and correspondingly, the potential is reduced around the potential maxima due to trapped electrons). Thus the ions accumulate in the trap until

$$
\Lambda_{i}=4 V_{s} \epsilon / r_{b} \text { (in cgs units) }
$$

and at this point the trap disappears, the ions exit to the cathode, and the electrons to the collector, as fast as they are produced.

As we have seen in the last section, the build up of ion charge in the unrippled beam causes a potential drop, which gives rise to an average field $V_{a x} / d$. A question is whether this electric field can cancel the maximum ripple induced electric field, and thereby eliminate the maxima and minima which trap the electrons and ions. The condition, from (16) and (17) is

$$
V_{a x} / d>2 V_{s} k\left(\epsilon / r_{b}\right)
$$

For either the CWI or the K376, the pressure required by (19) is much higher than the operating pressure, so we do not expect the oscilliatory nature of the ripple potential to be washed away by the dc ion flow in the unrippled beam.

The picture of ion noise from a scalloped beam then is the following. The ions and electrons build up in their traps, due to beam and avalanche ionization, and as they do, the beam voltage and microwave phase gradually change in response to this. However, at some point the traps are filled and additional ions generated, and maybe the trapped ions as well drain right to the cathode. Furthermore, as the ion current to the cathode varies, the focusing of the beam is also affected and therefore, so is $\epsilon$. At this point, the beam and microwave parameters are reset to their initial values, and a relaxation oscillation is set up.

Smith's work on the K376 also saw similar properties regarding ion current oscillation, but he did not do the careful optimization to eliminate oscillation. He saw high frequency ion current fluctuations, at frequencies from a few $\mathrm{kHz}$ to tens of $\mathrm{kHz}$. Since his pulses were only a millisecond long, he could not have seen the low frequency oscillations of Fig. 1. However, since his gas pressure was much higher, the ionization occurred faster and the frequency would be correspondingly higher.

Let us work out the basic period for these ion current or microwave phase variations. We begin with Smith's results because his background pressure is high enough that one does not have to worry about where the neutrals come from. To consider the ionization, one needs to consider ionization both by the primary beam electrons, and also by the secondaries. However, now these secondary electrons are assumed to be trapped below the potential maxima and become a separate fluid of trapped electrons. The trapping potential, for a $10 \%$ beam scallop is over $100 \mathrm{~V}$, just about the peak of the ionization cross section. Thus, even if an electron or ion is produced at rest, as it accelerates in the trapping potential, it gets energies of order $100 \mathrm{eV}$. Thus we consider the trapped particles to be a separate fluid as in Section III.

We rewrite the equation for the line charge density $\Lambda$, of secondary electrons or ions in the trap (6) as

$$
\frac{d \Lambda}{d t}=\sigma_{i} I N+\alpha_{i} \Lambda N
$$

where $\alpha_{s}$ is the ionization rate for the secondary electrons, $\alpha_{s}=\sigma_{o}\left(T_{e} / m\right)^{1 / 2}$, the peak ionization cross section times the thermal velocity of the electrons in the traps. We take $\alpha_{s}=1.3 \times 10^{-7} \mathrm{~cm}^{3} / \mathrm{s}$. The first term on the right of (20) is the direct ionization from the beam. The solution of (20), if $n=0$ at $t=0$ is

$$
\Lambda=\frac{\sigma_{i} I}{\alpha_{s}}\left(\exp \alpha_{s} N t-1\right) .
$$

The electron and ion density then build up according to (21). However, the line density can only build up to a maximum value given by (18). Thus, if the trapped density starts out as zero, the traps all dump in a time given by

$$
N t(\text { dump })=\alpha_{s}^{-1}\left[\ln \left(\frac{4 V_{s} \epsilon \alpha_{s}}{r_{b} \sigma_{i} I}+1\right)\right]
$$


where (22) is expressed in cgs units. From the known period of the ion oscillation, (22) can be used to give an estimate of the gas pressure in microwave circuit.

Assuming $\epsilon / r_{b}=0.1$, so that the electrons trapped in the ripples have about $100 \mathrm{eV}$ energy, we find

$$
N t(\text { dump })=5.4 \times 10^{6} \quad \text { for the CWI }
$$

and

$$
N t(\mathrm{dump})=6 \times 10^{6} \text { for the } \mathrm{K} 376 .
$$

In each case, avalanche ionization is somewhat more important than beam ionization.

Now let us review the measurements. Smith's main results were for pressures between $4 \times 10^{-6}$ and $1.5 \times 10^{-5}$. Shown in Fig. 10 are Smith's measurements of ion current as a function of time and pressure. The period is about $50 \mu$ s and it is not necessarily constant during the pulse. It is not easy to discern a dependence of period on pressure, but Smith seems to think that the period is generally a decreasing function of pressure. The pressure predicted by (23b) is about $3 \times 10^{-6}$ torr, about a factor of three below what is measured. It may be that in Smith's beam, which is probably not well matched after all his modifications to the experiment, the $\epsilon$ may be considerably larger.

We now turn to an analysis of the CWI. If the ripple amplitude is about $10 \%$, then the potential well can confine about $10 \%$ of the beam current density according to (18). However, one immediate problem is that for the very small radius beam and low pressure of the CWI, if the background gas (at an assumed pressure of $10^{-8}$ ) is fully ionized, it can contribute at most a few percent of the ions necessary to fill the trap. Thus recycling and resupplying of the neutrals must be playing a very crucial role in the noise in the ion CWI. If the neutrals recycle from the wall of the tube, which has a radius of only $1.5 \mathrm{~mm}$, then if they enter the tube at the sound speed at room temperature, the time for recycling from the walls is only $4 \mu \mathrm{s}$. Using the measured $10 \mathrm{~ms}$ dump time, we find that (23a) predicts a gas pressure of about $2 \times 10^{-8}$ torr, which is reasonably close to what is measured by the ion pumps. However, as we will see in Section VIII, ionization can reduce the pressure, and thereby it ought to reduce the frequency of the relaxation oscillation.

Let us finally comment on an aspect of Fig. 4. As the current in the input solenoid varies, the frequency can increase to over a kilohertz. This seems to imply that the pressure, at least near the input, also increases by about an order of magnitude. Furthermore, it implies that potential ripples near the input are more important than those further down. This is consistent with the arguments we made in Section II. Namely, if the velocity spread on the beam were greater than what is calculated by the gun electron gun simulations, the ripples further down stream would phase mix away. Indeed a velocity spread of only $6 \%$ would be sufficient to wash away the ripples at the beam exit of the CWI.

To summarize, the ion current oscillations that Smith observes, at least at high pressure, appear to be reasonably consistent with the model of filling and dumping of ion traps, particularly if the beam has a large ripple amplitude. However, the phase noise observed in the CWI appears to be consistent with this model only if the background gas density is maintained at its initial value in the presence of high ionization.

One additional conclusion which could be important is the following. The ionization from the secondary trapped electrons relies on the fact that the potential barrier formed by the beam scallops is large compared to the ionization potential. If a sufficiently well matched beam could be produced, so that the potential in the scallops is comparable to or less than the ionization potential, say a potential of $20 \mathrm{eV}$ or less, then the trapped secondaries could not further ionize the gas, and the wells would fill only by ionization by the primary beam, or by untrapped secondaries a slower process.

\section{The Penning TraP}

Now consider a potential like that shown in Fig. 5(c). Here a depressed collector is used, so as to increase the microwave efficiency by recovering a portion of the beam energy. In a uniform magnetic field, the secondary electrons now cannot get to either the collector or cathode; they are trapped in the tube body. This configuration is a Penning trap. This is an electron trap in which the electrons are confined radially by the magnetic field, and are confined axially by two large negative potentials at the ends. It is important to realize that in an azimuthally symmetric configuration, a thermal equilibrium exists for the trapped electron plasma. This is an equilibrium of the rigid rotor type whose electron distribution function is given by

$$
f(r, V)=\frac{n_{o}}{\left(\frac{2 \pi T}{m}\right)} \exp -\left(\frac{H-\omega P_{\theta}}{T}\right)
$$

where $H$ is the electron energy $H=1 / 2 m v^{2}-e \phi(r, z)$ and $P_{\theta}$ is the canonical momentum in the $\theta$ direction, $P_{\theta}=$ $m v_{\theta} r-e A r / c$, where $A$ is the vector potential. For a uniform field, $A=r B / 2$. Therefore, the electron density is give by

$$
n(r, z)=n_{o} \exp \frac{e \phi-m \omega\left(\Omega_{c}-\omega\right) r^{2} / 2}{T}
$$

where $\Omega_{c}$ is the electron cyclotron frequency. The density corresponds to that of a Maxwellian but with an additional effective potential proportional to $r^{2}$. In other words, the magnetic field gives the potential of a uniform background of positive charge. The self-consistent electrostatic potential $\phi$ may be calculated by inserting (25) into the right-hand side of Poisson's equation. Two-dimensional (2-D) self-consistent solutions have been found numerically by Prasad and O'Neil [10]. For the case of low temperature (or more precisely, a plasma dimension large compared to a Debye length $\lambda_{D}$ ), the density is only non zero if

$$
e \phi=\frac{m}{2} \omega\left(\Omega_{c}-\omega\right) r^{2}
$$

Relating the potential to the constant electron density, and using (26) we find

$$
\omega\left(\Omega_{c}-\omega\right)=\omega_{p e}^{2} / 2
$$




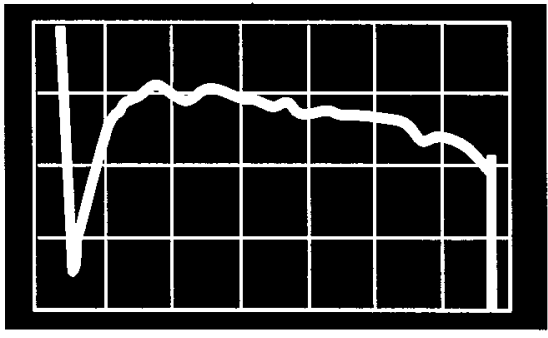

(a)

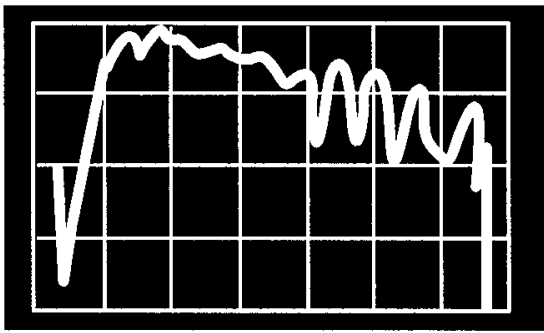

(c)

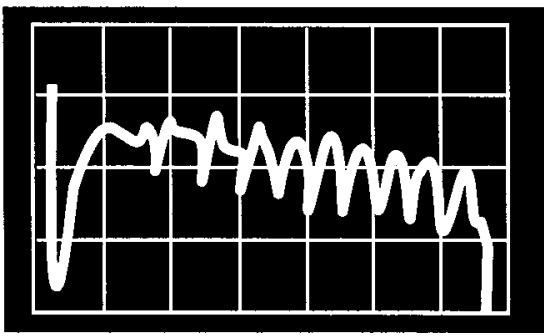

(e)

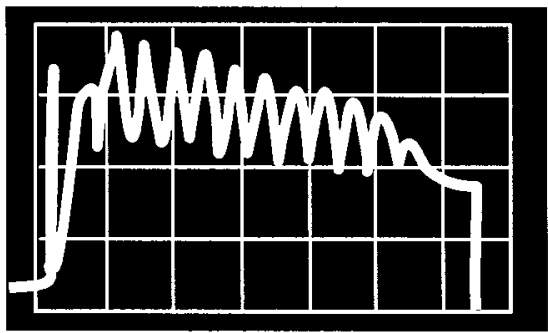

(g)

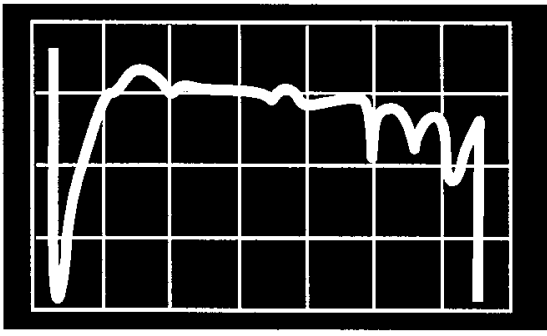

(b)

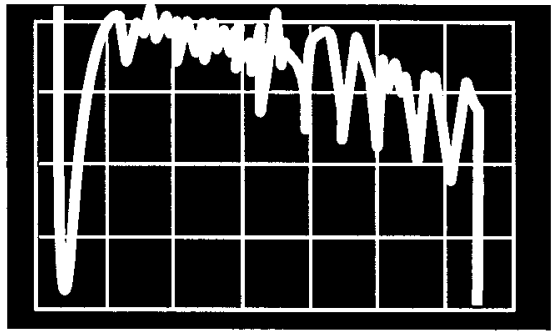

(d)

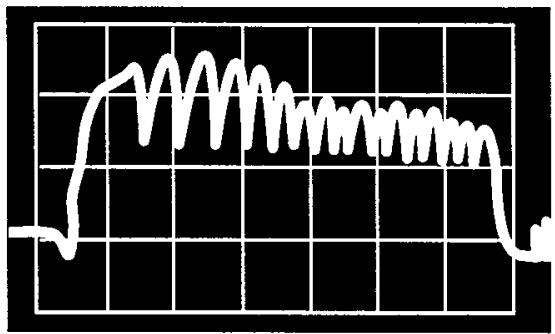

(f)

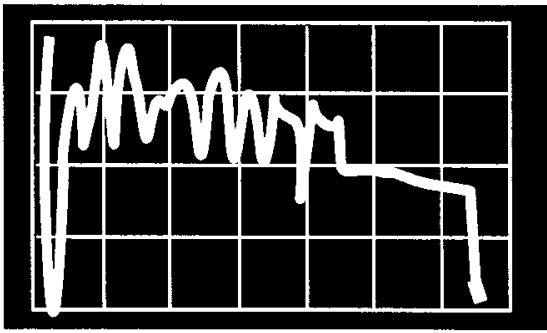

(h)

\section{ION CURRENT OSCILLATIONS \\ NORMAL BEAM CURRENT \\ COLLECTOR EARTHED \\ HORIZ: $100 \mu \mathrm{s} / \mathrm{div}$ \\ VERT: a-d $220 \mu \mathrm{a} / \mathrm{div}$$$
\text { e-h } 550
$$

Fig. 10. Plot of ion current as a function of time and at different pressures in Smith's experiment. Notice that it typically takes a certain time for the ion current to reach its equilibrium value, and then there is an oscillation about this value.

where $\omega_{p e}$ is the electron plasma frequency. The maximum electron density for which radial confinement exists is given by

$$
\left.\omega_{p e}^{2}\right|_{\max }=\frac{\Omega_{c}^{2}}{2}
$$

and this is the Brillouin condition. At the edge of the plasma there is a Debye length sheath at which the density falls to zero, and its shape is determined by the potential on the wall of the Penning trap and by the plasma density. Also, according to (26), the maximum radius of the plasma is determined by the fact that the maximum potential in the plasma must be below the confining potential.

The point is that the collisionless, azimuthally symmetric electron plasma in the Penning trap is in thermal equilibrium, 


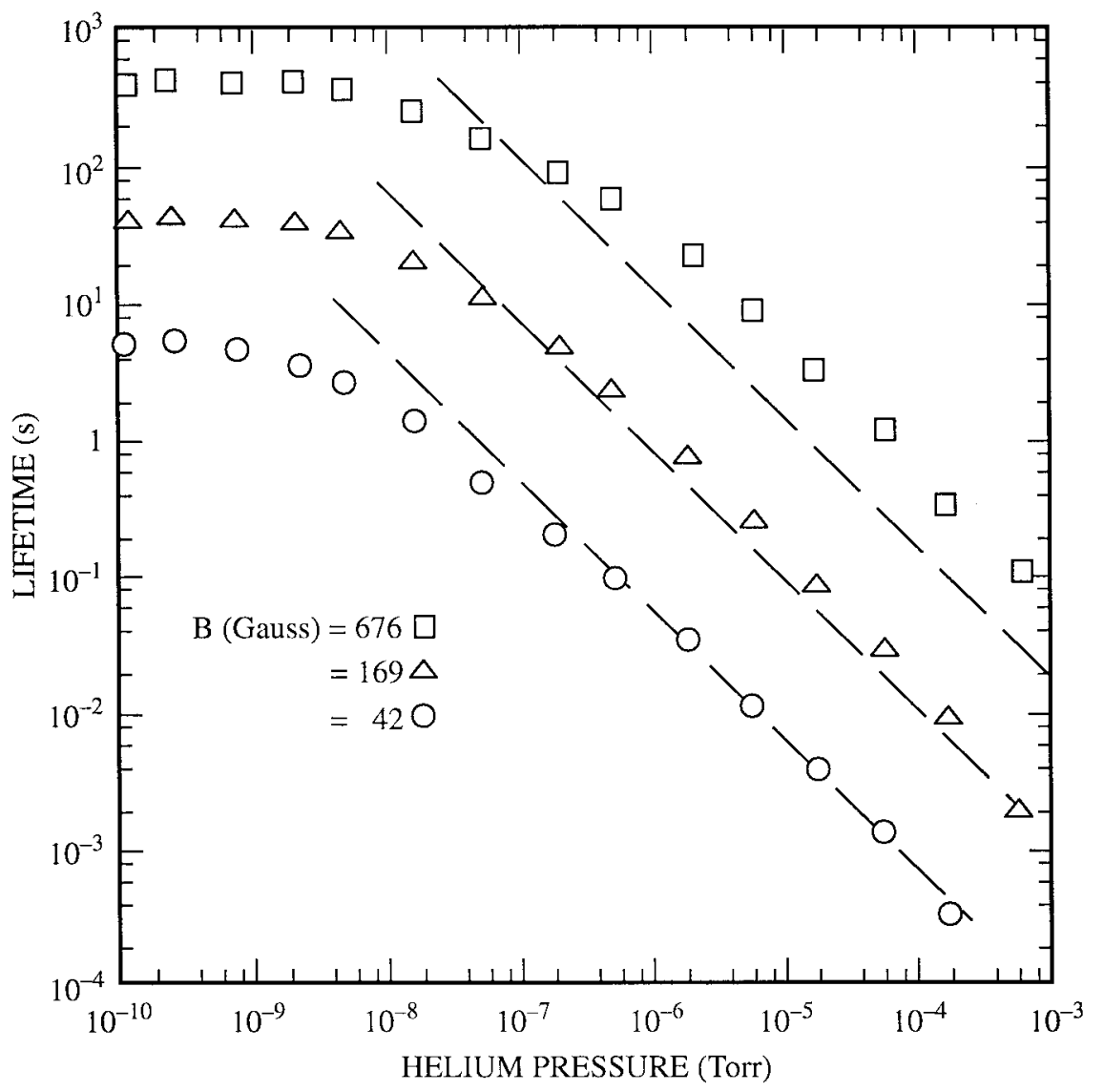

Fig. 11. Measured confinement time in a Penning trap sketched from [12].

which is unusual for a plasma. It is well known that there is no thermal equilibrium for a magnetically confined plasma containing both positive and negative charges. In practice, these pure electron plasmas can be contained for very long times. The group at the University of California at San Diego (UCSD) over about the past two decades have studied these plasmas and have in fact found extremely long lived confined nonneutral plasmas. The confinement time, at least for pressures above about $10^{-8}$ torr was determined by Malmberg and Driscoll [11] to be governed by collisions with the background neutrals.

Theoretically, this is simple to understand. If collisions with the background neutrals are present, but the electron neutral collision frequency $\nu$ is much less that the electron cyclotron frequency, and electron inertia is neglected, the electron momentum equations in the $r$ and $\theta$ directions become

$$
\begin{aligned}
\Omega_{c} V_{\theta}-\frac{e E_{r}}{m} & =0 \\
-\Omega_{c} V_{r}-\nu V_{\theta} & =0
\end{aligned}
$$

so that the azimuthal velocity is $c E / B$ and radial velocity is $v_{r}=c \nu E_{r} / \Omega_{c} B$. The radial electric field is the field from the confined charge. This charge might be either the beam charge or the confined plasma charge. We assume for now that the beam charge is dominant. In this case, for the CWI, the $E \times B$ azimuthal drift velocity of the electrons is about 6.5 $\times 10^{8} \mathrm{~cm} / \mathrm{s}$, that is an electron energy in excess of $100 \mathrm{eV}$. The electron momentum exchange collision frequency is $N \sigma v$ where $\sigma$ is the momentum exchange collision cross section and $v$ is the electron velocity. The confinement time is then $\tau=a /{ }^{2} v_{r}$ and it scales as

$$
\tau \propto B^{2} / P .
$$

Measured values of the confinement time compared with theory for a range of magnetic fields and pressures from (10) are sketched in Fig. 11. The largest magnetic fields in the experiment were only 676 Gauss, considerably smaller than in the CWI. Thus, at the pressures these tubes operate at, the lifetime of the electron confined in the Penning trap can be many minutes or even an hour or more.

Now let us work out a simple equation for the evolution of electron density in the trap assuming the density and radius are small enough that the electrons are radially and axially confined. The Penning trap in the CWI is different from those in the UCSD experiments because the magnetic field is reduced before the field lines enter the region of the depressed potential. Thus not all electrons produced by the beam are trapped. Some secondaries, those produced along the outer field lines, strike the wall and never make it into the depressed collector region. The beam electrons, on the other hand, have sufficient energy that they are nonadiabatic, and make it to the collector even though they have to cross field lines to do so.

To write an equation for the number density of trapped electrons, we will simplify the system somewhat and neglect avalanche ionization, but assume that the secondaries, as they 
bounce around on their confined orbits, generate on the average of a total three electrons, consistent with Bethe's formula and an assumed energy loss of about $30 \mathrm{eV}$ for each pair production (see Appendix A). As we have seen in the last section, considering the ripple trapped electrons as a fluid undergoing avalanche ionization, we found that the ion period estimate was consistent with beam ionization just slightly less important than direct beam ionization. Thus the neglecting avalanche ionization, but increasing beam ionization so it is consistent with classical estimates should be a reasonable approximation.

The electron production rate is then $3 \sigma_{i} I N / \pi e r_{b}^{2}$. The loss rate is $2 n v_{r} / r$. As we have seen, $v_{r}=\left(\nu / \Omega_{c}\right) c E_{r} / B$. The collision frequency is $N \sigma m v_{\theta}$, where $\sigma_{m}$ is the electron neutral momentum exchange collision frequency, typically about $10^{-15} \mathrm{~cm}^{2}$ for electron energy below about a few hundred $\mathrm{eV}$. The theta velocity is just the EXB drift $c E_{r} / B$. The next thing is a calculation of the electric field. In the region of the Penning trap, the field is generated by both the trapped electrons with density $n_{p}$ and the beam electrons with density $n_{b}$. As a function of radius, the electric field is given by $E_{r}=2 \pi e\left(n_{p}+n_{b}\right) r$.

This analysis leads to an equation for the trapped charge density

$$
\frac{d n}{d t}=\frac{3 \sigma_{i} I N}{e \pi r_{b}^{2}}-\frac{2 n}{r} \frac{N \sigma_{m} c^{2}}{\Omega_{c} B^{2}}(2 \pi e r)^{2}\left(n_{p}+n_{b}\right)^{2}
$$

where $r$ is the radius of the plasma in the trap. It is the radius of the last magnetic field line that makes it through the depressed collector. We define this as $r=\zeta r_{b}$. The factor $\zeta$ is less than unity, perhaps about 0.25 if the magnetic field at the depressed collector is 1/16th of the field in the microwave tube. Equation (31) gives an equilibrium density which solves the equation

$$
\eta(\eta+1)^{2}-A=0
$$

where $\xi=n_{p} / n_{b}$ and

$$
A=\frac{3 \sigma_{i}}{\sigma_{M}} \frac{I \Omega_{c} B^{2}}{\zeta r_{b}^{3} c^{2}\left(2 \pi e n_{b}\right)^{3}}
$$

For positive $A$, (32) has one real root and it is positive. In the limit of very small $A$, it is $\eta=A$, in the limit of very large $A$, it is $\eta=A^{1 / 3}$. For the CWI parameters we have been using $A=0.24 / \zeta$. For our assumed value of $\zeta=1 / 4, A$ is about equal to one, and the solution of (32) is approximately $\eta=0.5$. Thus the predicted plasma density is about one half of the beam density, and confined electron plasma extends up to about on quarter of the beam radius.

Let us determine the change in beam voltage from the confined plasma. Since the electron plasma has a radius smaller than the beam density, we will calculate the change in potential at the edge of the beam with and without the confined electron plasma. Assuming both the beam and plasma density are uniform up to $r_{b}$ and $\xi_{r b}$ (where $\xi=0.25$ ), respectively, we find the change in potential at the beam edge generated by the plasma is

$$
\Delta \phi=2 \pi e n_{p}\left(\xi r_{b}\right)^{2} \ln \left(a / r_{b}\right)
$$

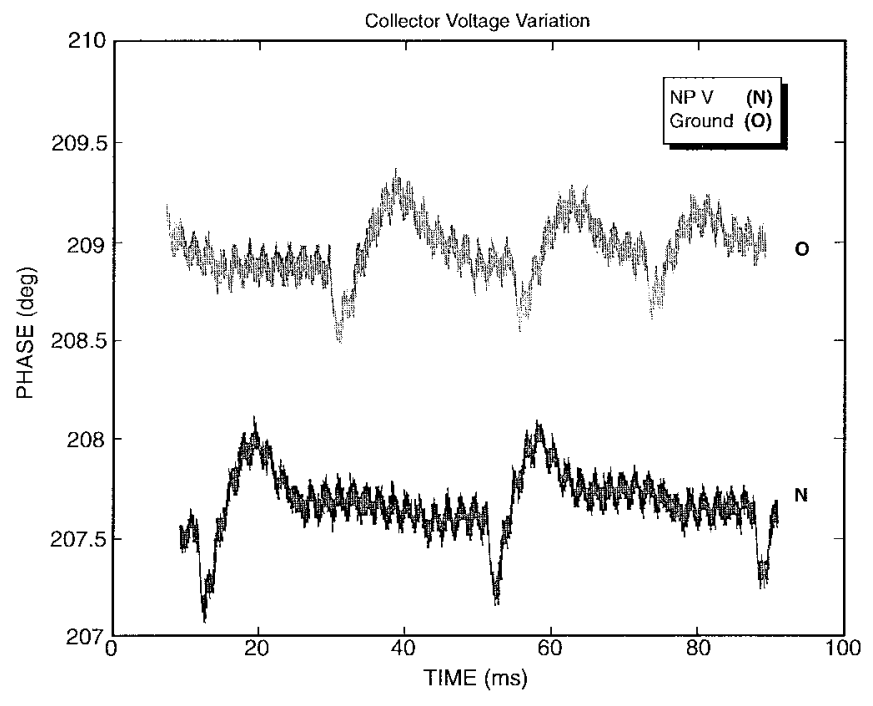

Fig. 12. Output phase of the CWI with and without the depressed collector.

This gives an additional potential depression at the beam edge of $10 \mathrm{~V}$, or a fractional voltage depression of $5 \times 10^{-4}$. This gives a relative phase change of one fourth of this value, as discussed in Section II. Hence the phase shift is predicted to be something over about $1^{\circ}$.

Experiments like this have been done at Crane, and they appear to roughly confirm this prediction. Fig. 12 shows ion noise measurements with the depressed collector on and off. (Actually the curve indicating the grounded collector actually has a collector with a small negative voltage, about minus 100 $\mathrm{V}^{2}$.) The two measurements show similar noise properties, more important, for the purpose of this discussion, is the measurement of ambient phase in the two. They are indeed about a degree and a half apart, and the phase was carefully followed as the collector was grounded.

Let us conclude by considering two additional aspects of the electrons in the Penning trap, the interaction of these electrons with the beam and microwaves. As regards the beam, there is the possibility that there could be a two stream instability between the beam and the confined plasma. Let us consider the CWI. If the Penning trap is filled to its maximum electron density $\left(\omega_{p e}^{2}=\Omega_{c}^{2} / 2\right)$, then the plasma density is about eight times the beam density. The maximum growth rate [12] of the instability is $\gamma=0.4 \omega_{p e}\left[n_{b} / n_{p}\right]^{1 / 3}$, and the group velocity of the fastest growing wave is $v_{g}=2 v_{b} / 3$. Since the instability is convective, the number of $e$-folding lengths is $L \gamma / v_{g}$. This is about 16 amplitude $e$ folds for the CWI. Depending on the initial noise level, this could well be a significant noise level and it could affect either the beam of the plasma. On the other hand, if the Penning trap is only slightly filled, the beam is the heavier fluid, the maximum growth rate is $0.4 \omega_{p b}\left[n_{p} / n_{b}\right]^{1 / 3}$, and the group velocity is one third the beam velocity. In this case there are about eight amplitude $e$-folding lengths in the microwave circuit. Possibly, this beam plasma instability could be an additional factor leading to axial loss of the trapped electron plasma.

Finally we consider the effect of the microwaves on the background plasma. The beam sets up a microwave signal 
by its interaction with the microwave circuit. This microwave circuit sets up a slow wave in the drift tube, and this slow wave takes energy from the beam. Typically, the longitudinal fields of the microwave trap the beam, so that the beam slows down to the phase velocity and also acquires some thermal spread. Usually, the phase velocity is reasonably close to the beam velocity. For the sake of argument, let us consider a phase velocity which is $85 \%$ of the beam velocity. Then for the beam to be trapped by the microwave, the trapping width $v_{T}=2\left[e \phi / m_{k}\right]^{1 / 2}$ must be equal to about $15 \%$ of the beam velocity. Here $\phi$ is the longitudinal potential of the parallel microwave electric field and $k$ is the longitudinal wave number. For the microwave field to trap the main plasma, the trapping frequency would have to be about six times larger, or the power of the microwave field, which is proportional to $\phi^{2}$ would have to be larger by a factor of 1296 . Thus it seems very unlikely that the microwave field produced by the beamwave interaction will have a very large affect on a background electron plasma in the Penning trap.

While our calculation shows that the Penning traps in the CWI do not fill to very high density, an interesting speculation could be on what the effect would be on a CW gyrotron (or other gyro device). The magnetic fields and confining potentials are now much greater. Furthermore, beam electrons would normally adiabatic well into the depressed collector region. It could be that such a $\mathrm{CW}$ gyrotron with a depressed collector might have a very high density of trapped electrons.

\section{THE GAS SUPPLY}

Here we investigate very briefly the question of gas supply in the CWI. Let us say that in the microwave circuit part of the tube, there is a source of gas $S_{g}$ and that it is pumped out at a rate $\zeta$. The maximum that $\zeta$ can be is the sound speed divided by the length of the tube, or about $2 \times 10^{3} \mathrm{~s}^{-1}$ for the CWI. If the background pressure is $10^{-8}$ torr, this means that $S_{g}$ is $6 \times 10^{11} \mathrm{~cm}^{-3} \mathrm{~s}^{-1}$ or less. Now let us look at the effect of ionization. Considering only beam ionization, for the 3 Amp beam in the CWI, the equation for the gas density is

$$
\frac{d N}{d t}=S_{g}-\zeta N-3 \times 10^{3} N
$$

Thus, taking the minimum value for ionization and the maximum value for pump speed, we see that beam ionization will significantly increase the neutral pump out rate. It seems likely that ionization will significantly deplete the neutrals (by a factor of two or more) if the gas source $S_{g}$ is unaffected by the beam. One intriguing possibility is that the gas source is in fact affected by the beam, and that the noisy tubes have a larger $S_{g}$ than the quite tubes.

\section{FUTURE THEORETICAL WORK}

Assuming that ion noise is a serious enough problem that significant resources will be committed to it, we consider here what directions future theoretical work might take. The main need, it seems, is for the development of numerical simulation schemes to examine more carefully the physics developed in Sections IV, V, and possibly VI. There are many levels of difficulty one could envision. The simplest would be a onedimensional (1-D) code in the axial coordinate. This would be a Monte Carlo style particle simulation. There would be some background gas and it would be ionized by the beam. The secondary electrons and ions and all their progeny would be followed axially in their self-consistent potential. The calculation of the potential would be extremely simple, (10) shows that it is just proportional to the total charge density. Thus the potential calculation is simpler than a solution of Poisson's equation. With a simulation like this, the 1-D ion drain to the cathode could be simulated with or without a depressed collector. Also the 1-D filling and dumping of potential buckets caused by beam scalloping could be simulated by simply modeling the beam scallops as externally imposed axial potential variations.

However, as with any such code that attempts to model the microphysics, there are difficulties regarding the disparate time scales. Some of the important time scales for such a simulation are enumerated in Table I. The fastest time scale is the inverse cyclotron frequency of about 20 ps. However, the simulation must proceed for times very long compared to the ionization time, or for at least $10 \mathrm{~ms}$ if the background pressure is $10^{-7}$ torr. This is nine orders of magnitude in time scale. Notice also that any electron or ion dynamic time scale is much faster than any of the processes measured in either Smith's or the CWI work. These experiments seem to be measuring mostly collisional processes.

Clearly, to simulate, the time scales must be very greatly compressed. One possibility is not to follow the electron gyro motion, but rather to follow only the guiding center, and allow perpendicular and parallel velocities to exchange with the various collision processes. However, this only increases slightly the minimum relevant time. However if the background pressure is artificially increased by perhaps four orders of magnitude, the ionization time is speeded up to about 1 $\mu \mathrm{s}$, and it might be possible to follow the electron bounces in the well and also the ionization and associated relaxation oscillation. However, one must be cautious not to artificially raise the background pressure to too high a value. If one does this, (19) shows that the ripple potentials may themselves be artificially washed out. Furthermore, (14) shows that in the normal potential profile, the equilibrium ion density will be greatly increased at high pressure. In fact at an artificially high pressure, the ion density may build to such a high value that radial confinement is lost, whereas in the physical tube, where the pressure is much lower, the ions are well confined radially. Also note that if the neutral density is artificially increased, electron electron collisions are much less important than electron neutral collisions (that is if the plasma electron density is $10^{10} \mathrm{~cm}^{-3}$, or $10 \%$ of the beam density), whereas in the physical problem, they are about as important as ionization.

Another possibility is to artificially increase the electron mass. In the theory, as developed up to now, the actual electron mass has played almost no role. The main assumption has only been that electrons move fast compared to the ions. Increasing the electron mass to perhaps one one hundredth, or even one tenth of the ion mass could preserve the essential physics of ion noise in microwave tubes, while making the simulation run much faster. 
TABLE I

Relevant Electron and Ion Time Scales

\begin{tabular}{|c|c|c|}
\hline Process & Formula & Time (sec) for CWI \\
\hline $\begin{array}{l}\text { Cyclotron } \\
\text { Motion }\end{array}$ & $\Omega_{\mathrm{c}}^{-1}$ & $2 \times 10^{-11}$ \\
\hline $\begin{array}{l}\text { Electron } \\
\text { Bouncing in } \\
\text { Scallops }\end{array}$ & $\left(k_{B} V_{e}\right)^{-1}$ & $4 \times 10^{-11}$ \\
\hline Ion Axial Bounce & $\left(\mathrm{k}_{\mathrm{B}} \mathrm{V}_{\mathrm{i}}\right)^{-1}$ & $2 \times 10^{-9}\left(\mathrm{M}_{\mathrm{p}} / \mathrm{M}\right)^{1 / 2}$ \\
\hline Ion Radial Bounce & {$\left[\mathrm{M} / 2 \pi \mathrm{n}_{\mathrm{b}} \mathrm{e}^{2}\right]^{1 / 2}$} & $3 \times 10^{-9}\left(\mathrm{M}_{\mathrm{p}} / \mathrm{M}\right)^{1 / 2}$ \\
\hline $\begin{array}{l}\text { Electron } \\
\text { Elastic } \\
\text { Collision }\end{array}$ & $\left(N \sigma_{p} V_{e}\right)^{-1}$ & $5 \times 10^{-11} / \mathrm{P}($ torr $)$ \\
\hline $\begin{array}{l}\text { Ion Neutral } \\
\text { Charge } \\
\text { Exchange }\end{array}$ & $\left(N \sigma_{x} V_{i}\right)^{-1}$ & $2 \times 10^{-9}\left(\mathrm{M} / \mathrm{M}_{\mathrm{p}}\right)^{1 / 2} / \mathrm{P}($ torr $)$ \\
\hline Avalancne Ionization & $\left(N \sigma_{i} V_{e}\right)^{-1}$ & $2.5 \times 10^{-10} / \mathrm{P}$ (torr) \\
\hline $\begin{array}{l}\text { Electron } \\
\text { Electron } \\
\text { Collisions }\end{array}$ & $3 \times 10^{5} \mathrm{~T}_{\mathrm{e}}{ }^{3 / 2} / \mathrm{n} \lambda$ & $3 \times 10^{7} / \mathrm{n}\left(\mathrm{cm}^{-3}\right)$ \\
\hline $\begin{array}{l}\text { Ion Ion } \\
\text { Collisions }\end{array}$ & $2 \times 10^{7} \mathrm{~T}_{\mathrm{i}}^{3 / 2}\left(\mathrm{M} / \mathrm{M}_{\mathrm{p}}\right)^{1 / 2} / \mathrm{n} \lambda$ & $2 \times 10^{9}\left(\mathrm{M} / \mathrm{M}_{\mathrm{p}}\right)^{1 / 2} / \mathrm{n}\left(\mathrm{cm}^{-3}\right)$ \\
\hline \multicolumn{3}{|c|}{$\begin{array}{l}\text { Notes: Electron and ion thermal energies are assumed to be } 100 \mathrm{eV} \text { characteristic of } \\
\text { ripple well depth, } \mathrm{M}_{\mathrm{p}} \text { is the proton mass, Coulomb logarithm is assumed equal to } 10 \text {, } \\
\text { Elastic, ionization and charge exchange cross sections typically as given in Ref. (6), ee } \\
\text { and ii collisions as given in Ref. (11), other parameters as quoted for the CWI. }\end{array}$} \\
\hline
\end{tabular}

A simulation code which treats the electrons as guiding centers, but contains all other processes has been developed for the NRL plasma processing program [13], and it could be adapted rather easily to the problem of ion noise in microwave tubes. In fact since the code of [13] treats the electron electron collision as a grid quantity, these collisions could be artificially speeded up also.

It would be important to include models for the atomic physics that are as accurate as possible. For a particular gas, it should certainly include reasonably accurate models for the ionization by the beam, as well as reasonable models for the energy distributions of the secondaries. The ionization of the gas by the secondaries is also very important for cases where they are confined, and possibly is important as well where they are unconfined. This should be followed as well.

Once one has a 1-D axial PIC/Monte Carlo simulation, the next obvious thing is to extend it to two dimensions. The calculation of the potential would now involve both the radial and the axial charge density. With a 2-D simulation, one could simulate the radial loss of confinement of the ions when their density becomes as large as the beam density, as well as the collisional radial flux of electrons.

A more complicated part, but a very important one is the gas recycling and resupply. Especially for the CWI, this appears to be playing a very important part. This would undoubtedly involve physical models for gas absorption and desorbtion from the walls, and the filling of the tube by the desorbed gas.

As is apparent from Fig. 3, in at least certain cases, the phase oscillation has the form of a relaxation oscillation where it snaps back very quickly. It seems as though in these cases, an instability may be occurring. There could be interactions between electrons and ions trapped in separate, but spatially overlapping traps, and at some critical density, this equilibrium could be unstable.

Finally, if one knows the ion drainage to the cathode, one can add specified positive charges and currents to the electron gun and focusing regions and see what effect this has on the 
production of the electron beam. It seems that this effect could be easily added to the e-gun simulations.

\section{CONCLUSIONS}

We have sketched certain elements of the theory of ion noise in linear microwave tubes. The measurements of ion current to the cathode in Smith's experiment seem to be reasonably consistent with theory, but knowledge of the ion mass would give further confidence. A very useful experiment would be to repeat Smith's experiment with a mass spectrometer behind the cathode to investigate what the ions are. The results of ion current oscillation in Smith's experiment also seem consistent with electrostatic traps generated by the beam ripples filling and dumping. The CWI ion noise experiments show a similar effect. In both cases, the relaxation oscillation time seems to be reasonably consistent with theory. The sensitivity of the ion noise in the CWI to solenoidal field and voltage may be result from a combination of the number of beam scallops which occur in the interaction region, as well as to the entrance conditions. If the beam has some thermal spread, entrance conditions would become more important. and this too seems to be at least qualitatively consistent with theory. We further speculate that the difference between quiet and noisy tubes may have to do with what the actual gas supply is in a running tube. Finally, in tubes with a depressed collector, the Penning traps do seem to fill up with electrons about as predicted. It is possible that gyrotrons or other gyro devices with depressed collectors may fill to a much higher density of secondary electrons.

\section{APPENDIX A}

\section{AN ApProximate Calculation of the IONIZATION} MULTIPLICATIVE FACTOR FROM IONIZATION BY SECONDARIES

The cross section for direct ionization by the beam is given in for instance Fig. 6 and in (4) at higher beam energy. As the beam traverses the gas it loses energy, and this energy goes into ionization, the energy of the secondaries, various excitations, and the recoil of the atom. The secondary electrons produced generally have a rather low energy distribution, but some fraction of them do have energy above the ionization energy; if the energy of the secondary is, say $100 \mathrm{eV}$, its ionization cross section is much greater than that of the beam electron. The number of secondaries produced by a primary is then determined experimentally, for instance by measuring the current, if a small electric field is imposed on a gas which contains a radioactive beta decay element which produces a beta at known rate and at known energy. Also the number is calculated theoretically by Monte Carlo calculations. A primary is followed as it ionizes and collides. Then the secondary and tertiary, etc. electrons are also followed until all slow down to the point where they have energy below the ionization energy. The simulation then keeps track of the total number of electrons produced. Very shortly, we will present here a very approximate calculation of this type.

A simple way to calculate the number of electron ion pairs produced per primary (i.e., beam electron) is to use the tabulated energies to produce an electron-ion pair for a primary of a given energy. These values are derived either from experiments or from Monte Carlo calculations. These are tabulated for instance by Christophorou [8] for many common gases. For typical diatomic gases, the energy to produce an electron ion pair is about $30 \mathrm{eV}$. Since the ionization energy is typically about 15 electron volts, beam ionization is rather efficient; typically about half the lost energy goes into ionization. One can then find the number of electrons produced by the beam by using Bethe's formula for energy loss of the beam in the gas [14]. By dividing the rate of energy loss per $\mathrm{cm}$ by the energy to produce an electron ion pair, one determines the number of electron ion pairs produced per $\mathrm{cm}$. Then by using the primary ionization cross section, (4), one determines the production rate from the primaries alone. The ratio is then the number of electrons produced per primary.

Bethe's formula for high energy electrons in oxygen [9], [10] is

$$
\frac{d E(\mathrm{eV} / \mathrm{cm})}{d x}=\frac{2 \times 10^{-15} N \ln 10 E(\mathrm{keV})}{E(\mathrm{keV})}
$$

whereas the rate of production by primaries is

$$
\frac{d n_{\text {pri }}}{d x}=N \sigma_{i}
$$

Taking the value of $30 \mathrm{eV}$ for electron ion pair production, we find that the total number of electrons produced per primary ionization is about 3.1 , or about 2.1 ionizations by the secondaries, tertiaries, etc.

We now perform an approximate calculation of the ionization by the secondaries as one might do in a Monte Carlo simulation. A secondary electron is produced with some energy distribution, and one clearly needs this to continue. One example is for Argon [15]. The relative distribution of secondaries for a primary electron of energy $E$ is denoted $S_{M}(E, T)$, where $T$ is the energy of the secondary electron plus $15.76 \mathrm{eV}$. A plot of $S_{M}(E, T)$, sketched from [18] is shown in Fig. 13. Here $R$ is $13.6 \mathrm{eV}$. An approximation to the plot, for a $10 \mathrm{keV}$ primary, is shown by the heavy lines. Notice that a constant value on the plot actually means that the distribution function is proportional to $T^{-2}$. The break in the curve in Fig. 13 is at about the ionization energy of argon, $15.76 \mathrm{eV}$, which we will call $E_{o}$ here. Thus as an approximate distribution function for the secondary energy, which we now call $E_{s}$, we have

$$
\begin{aligned}
& f\left(E_{s}\right)=1.6 E_{o} /\left(E_{s}+E_{o}\right)^{2} \quad E_{s}<E_{o} \\
& f\left(E_{s}\right)=0.4 E_{o} /\left(E_{s}+E_{o}\right)^{2} \quad E_{s}>E_{o}
\end{aligned}
$$

where the distribution function is now normalized to unity. Secondary electrons can generate additional ionization if $E_{s}>E_{o}$. Integrating the distribution function, we find that about $20 \%$ of the secondaries can give rise to additional ionization. Now let us calculate the effective ionization cross section from these secondaries. Fig. 6 shows that the ionization cross section has a broad maximum $\sigma_{o}$ of about 2 $\times 10^{-16} \mathrm{~cm}^{2}$ for energies of about $60 \mathrm{eV}$ to about $1 \mathrm{keV}$, and below $60 \mathrm{eV}$, the ionization cross section rises roughly linearly from zero to this maximum as the energy increases from $E_{o}$ to $60 \mathrm{eV}$ (about $4 E_{o}$ ). If we take the approximation 


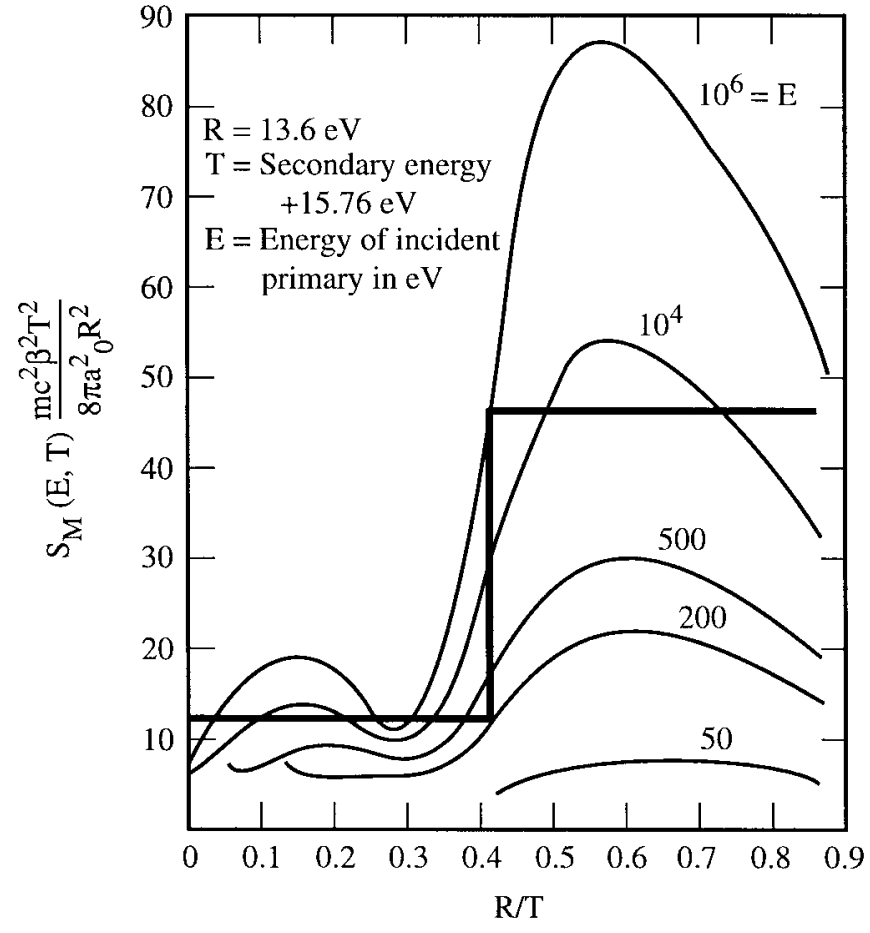

Fig. 13. Energy distribution of secondary electrons for ionization of argon by a fast electron.

to the distribution function given in (C3) and average the cross section, this average cross section from the secondaries turns out to be about $\sigma_{s}=0.08 \sigma_{o}$, or about $1.6 \times 10^{-17} \mathrm{~cm}^{2}$. This is about three times the primary ionization cross section of about $5 \times 10^{-18} \mathrm{~cm}^{2}$ for a $20 \mathrm{keV}$ electron. Thus from this simple calculation, we find that the secondaries are responsible for about three times the ionization of the primaries. The total number, from our previous calculation from Bethe's formula, was about three total electrons produced for each primary (including the primary). Thus our calculation gives reasonable qualitative agreement.

\section{APPENDIX B}

\section{THE INTEGRAL EQUATION FOR THE STEADY STATE ION CURRENT AND ITS SOLUTION}

We assume that the ion flow is collisionless and that there is an ion source which is given by $G_{i}$ which is uniform in space. Here, $G_{i}$ is $I \sigma_{i} N$. Each region of length $d x$ produces ions which accelerate from their origin to the cathode. For convenience, we consider positive ion velocities, so in the configuration for this appendix, the cathode is on the right. Hence at each position, the ion density is the sum of all ion beamlets produced to its left. Hence the ion line density at position $z, \Lambda_{i}(z)$ is given by

$$
\Lambda(x)=\int_{0}^{x} d y \frac{G_{i} d y}{\sqrt{\frac{2 e K}{M}[\Lambda(y)-\Lambda(x)]}}
$$

where $x=0$ is the left most position at which ions are produced, and where we have used the relation between potential along the axis and linear charge density, (9) and
(10). Equation (A1) clearly only has solutions in which $\Lambda$ is a monotonically decreasing function of $x$. As discussed in Section IV, the beam density is not included in (B1) because it is uniform axially, and the space charge of the secondary electrons is not included because it is assumed to be much less than the ion charge density, due to their greater velocity.

To solve (B1), change the independent variable on the righthand side from $y$ to $\Lambda(y)$. Then, (B1) takes the form

$$
\lambda=\frac{Q}{\sqrt{\Lambda_{0}}} \int_{1}^{\lambda} \frac{P\left(\lambda^{\prime}\right) d \lambda^{\prime}}{\sqrt{\lambda^{\prime}-\lambda}}
$$

where $Q=G \Lambda_{o}^{-1}(2 e K / M)^{-1 / 2}, \Lambda_{o}=\Lambda(x=0), \lambda=\Lambda / \Lambda_{o}$, and $P(\lambda)=d x / d \lambda$. Thus, (B2) is an integral equation for $P(\lambda)$, and thereby for the axial profile of the potential. It is an equation of the Abel type. As a solution, try some function of the form

$$
P(\lambda)=\frac{\Xi(\lambda)}{\sqrt{1-\lambda}}
$$

where $\Xi$ is a simple function of $\lambda$. Inserting (B3) into (B2), the radical in the denominator is a quadratic which is positive between $\lambda<\lambda^{\prime}<1$, the range of the integration. By forming a simple table of the integral of

$$
\Lambda=\int_{1}^{\lambda} \frac{\Xi\left(\lambda^{\prime}\right) d \lambda^{\prime}}{\sqrt{\left(\lambda^{\prime}-\lambda\right)\left(1-\lambda^{\prime}\right)}}
$$

for various simple functions $\Xi$, we find that

$$
\Gamma=-\pi \text { for } \Xi=1
$$

and

$$
\Gamma=-(\pi / 2)[1+\lambda] \text { for } \Xi=\lambda .
$$

Thus the solution to (B2) is

$$
\frac{d x}{d \Lambda}=-\frac{\Lambda_{o}^{3 / 2}}{\pi Q} \frac{2 \frac{\Lambda}{\Lambda_{o}}-1}{\sqrt{1-\frac{\Lambda}{\Lambda_{o}}}} .
$$

Equation (B6) shows that $d \Lambda / d x=0$ at $\Lambda=\Lambda_{o}$, the furthest point from the cathode. As the ions accelerate, the derivative becomes singular at $\Lambda / \Lambda_{o}=1 / 2$. This presumably is the position where the solution matches onto some different solution where $\Lambda$ varies much more quickly in $x$. In other words, the singularity at $\Lambda / \Lambda_{o}=1 / 2$ marks the position where solution in the drift tube joins onto the solution in the gun region. This then confirms the estimate in Section II, where it was assumed that at the cathode end, the ion charge density is just half of what it is at the collector end of the drift tube. In terms of the parameters of the ion source and geometry, (B6) shows that the maximum ion line charge density near the collector end of the tube is given by

$$
\Lambda_{o}=\left[\frac{9 \pi^{2} G_{i}^{2} d^{2}}{16\left(\frac{e}{M}\right) K}\right]^{1 / 3} .
$$

This is very close to the rough estimate derived in Section II. Smith points out that the total ion charge contained can be 
obtained by numerically integrating $\Lambda(x)$ along the tube. He finds the result

$$
Q_{i}=0.9 \Lambda_{\circ} d
$$

\section{ACKNOWLEDGMENT}

The author wishes to thank D. Chernin and H. Freund of SAIC, Y. Y. Lau of the University of Michigan, B. Levush and R. Fernsler of NRL, and T. Antonsen of University of Maryland, for a number of useful discussions. The author especially thanks S. Gilmour for discussions concerning the nature of the Penning trap, and whether electrons generated in the outer radii of the beam make it into the depressed collector, and D. Thelan of TSC for helping with interpretation of the Crane data and allowing its reproduction here.

\section{REFERENCES}

[1] J. K. Smith, "Ion drainage in high power klystrons," Ph.D. dissertation, Cambridge Univ., U.K., July, 1972.

[2] D. Thelan, private communication, Sept. 1998.

[3] R. English and W. Funk, CWI TWT Ion Noise Study Report, Technical Service Corporation, Bloomington, IN 47404, Document 0159, Mar., 1998.

[4] S. Gilmour, private communication, June 1998.

[5] D. Thelan, to be published.

[6] Y. Y. Lau and D. Chernin, and W. Manheimer, "A note on noise in linear beam microwave tubes," IEEE Trans Electron Devices, submitted for publication.

[7] S. C. Brown, Basic Data of Plasma Physic. Cambridge, MA: MIT Press, 1967.
[8] L. Christophorou, Atomic and Molecular Radiation Physics. New York: Wiley Interscience, 1971, p. 37.

[9] T. Majeed and D. Strickland, "New survey of electron impact cross sections for photoelectron and auroral energy loss calculations," J. Phys. Chem. Ref. Data, vol. 26, no. 2, pp. 335-349, 1997.

[10] S. Prasad and T. O'Neil, "Finite length thermal equilibria of a pure electron plasma column," Phys. Fluids, vol. 22, p. 278, 1979.

[11] J. Malmberg and C. Driscoll, "Long time confinement of a pure electron plasma," Phys. Rev. Let., vol. 44, p. 654, 1980.

[12] D. L. Book, NRL Plasma Formulary, Naval Research Lab., Washington, DC, 1983.

[13] M. Lampe, G. Joyce, W. Manheimer, and S. Slinker, "Quasineutral particle simulation of magnetized plasma discharges: General formalism and application to ECR discharges," IEEE Trans. Plasma Sci., vol. 26, pp. 1592-1609, Dec. 1998.

[14] J. Jackson, Classical Electrodynamics. New York: Wiley, 1975, ch. 13

[15] J. Bretagne, G. Calledo, M. Legentil, and V. Puech, J. Phys. D, Appl. Phys., vol. 19, p. 761, 1986.

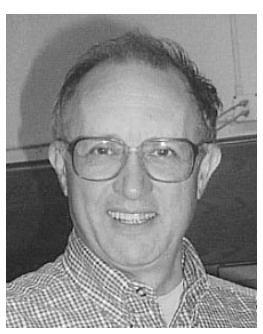

Wallace M. Manheimer (M'85-SM'86-F'93) received undergraduate and graduate degrees at Massachusetts Institute of Technology, Cambridge, MA

He has been with the Naval Research Laboratory (NRL), Washington, DC, since 1970, and he currently serves as Senior Scientist for Fundamental Plasma Processes. His interests include principally plasma physics, electron beams, and microwave tubes. During his tenure at NRL, he has had two external sabbaticals, one as a Visiting Scientist at Culham Laboratory, at Abingdon, England in 1977-1978 and one as a Visiting Professor of Physics at Moscow State University, Russia, in 1995, and one internal sabbatical in NRL's Radar Division in 1990. 